\title{
THE SEMIDISCRETE FILTERED BACKPROJECTION ALGORITHM IS OPTIMAL FOR TOMOGRAPHIC INVERSION*
}

\author{
ANDREAS RIEDER ${ }^{\dagger}$ AND ADEL FARIDANI ${ }^{\ddagger}$
}

\begin{abstract}
The filtered backprojection algorithm is probably the most often used reconstruction algorithm in two-dimensional computerized tomography. For a semidiscrete version in the parallel scanning geometry we prove optimal $L^{2}$-convergence rates for density distributions in Sobolev spaces. Additionally we show $L^{2}$-convergence without rates when the density distribution is only in $L^{2}$. The key to success is a new representation of the filtered backprojection which enables us to apply techniques from approximation theory. Our analysis provides further a modification of the SheppLogan reconstruction filter with an improved convergence behavior. Numerical experiments in the fully discrete setting reproduce the theoretical predictions.
\end{abstract}

Key words. Radon transform, tomography, filtered backprojection algorithm, reconstruction filter

AMS subject classification. 65R20

PII. S0036142902405643

1. Filters in tomography. Tomographic reconstruction means finding a density distribution $f$ from all its line integrals $g=\mathbf{R} f$. Here, $\mathbf{R}$ denotes the Radon transform,

$$
\mathbf{R} f(s, \vartheta):=\int_{L(s, \vartheta) \cap \Omega} f(x) \mathrm{d} \sigma(x),
$$

mapping a function to its integrals over the lines $L(s, \vartheta)=\left\{\tau \omega^{\perp}(\vartheta)+s \omega(\vartheta) \mid \tau \in \mathbb{R}\right\}$, where $s \in \mathbb{R}, \omega(\vartheta)=(\cos \vartheta, \sin \vartheta)^{t}$, and $\omega^{\perp}(\vartheta)=(-\sin \vartheta, \cos \vartheta)^{t}$ for $\left.\vartheta \in\right] 0, \pi[$. This parameterization of lines gives rise to the parallel scanning geometry. The Radon transform $\mathbf{R}$ maps $L^{2}(\Omega)$ boundedly to $L^{2}(Z)$, where $\Omega$ is the unit ball in $\mathbb{R}^{2}$ centered about the origin and $Z$ is the rectangle $Z=]-1,1[\times] 0, \pi[$.

Analytically, tomographic reconstruction is represented by the inversion formula

$$
f=(2 \pi)^{-1} \mathbf{R}^{*} \Lambda g,
$$

where the backprojection operator $\mathbf{R}^{*}: L^{2}(Z) \rightarrow L^{2}(\Omega)$ is the adjoint to $\mathbf{R}$,

$$
\mathbf{R}^{*} \Phi(x):=\int_{0}^{\pi} \Phi\left(x^{t} \omega(\vartheta), \vartheta\right) \mathrm{d} \vartheta .
$$

Formally, $\Lambda$ is the square root of the one-dimensional Laplacian $-\Delta: \Lambda=(-\Delta)^{1 / 2}$. In (1.1), $\Lambda$ acts on the variable $s$ of $g$. For a proof of (1.1) see, e.g., Natterer [14].

Due to the compactness of $\mathbf{R}$ the reconstruction of $f$ from noisy Radon data $g$ by (1.1) is unstable ( $\Lambda$ amplifies high frequencies). A stable algorithm of tomographic reconstruction is therefore based on

$$
f \star e_{\gamma}=\mathbf{R}^{*}\left(v_{\gamma} \star_{s} g\right), \quad e_{\gamma}=\mathbf{R}^{*} v_{\gamma},
$$

*Received by the editors April 15, 2002; accepted for publication (in revised form) October 23, 2002; published electronically May 22, 2003.

http://www.siam.org/journals/sinum/41-3/40564.html

${ }^{\dagger}$ Institut für Wissenschaftliches Rechnen und Mathematische Modellbildung, Universität Karlsruhe, 76128 Karlsruhe, Germany (andreas.rieder@math.uni-karlsruhe.de).

$\ddagger$ Department of Mathematics, Oregon State University, Corvallis, OR 97331 (faridani@math. orst.edu). The research of this author was supported by NSF grant DMS-9803352. 
where $\star$ denotes convolution and $\star_{s}$ denotes convolution with respect to the variable $s$. In (1.2), $e_{\gamma}(x)=e(x / \gamma) / \gamma^{2}, \gamma>0$, and $e=e_{1}$ is a mollifier, that is, a smooth function with normalized mean value. Thus, $f \star e_{\gamma}$ is a smoothed or mollified approximation to $f$. The function $v=v_{1}$ is called the reconstruction kernel or reconstruction filter which is independent of the angle $\vartheta$ for radially symmetric mollifiers (which we assume in what follows). Note that $v_{\gamma}(s)=v(s / \gamma) / \gamma^{2}$. By the inversion formula (1.1) we can compute the reconstruction kernel from a mollifier $e$ :

$$
v=\frac{1}{2 \pi} \Lambda \mathbf{R} e .
$$

The convolution $v_{\gamma} \star_{s} g$ realizes a low pass filtered version of $\Lambda g /(2 \pi)$.

A straightforward discretization of (1.2) together with an interpolation step yields the filtered backprojection algorithm (FBA) which is the most frequently used algorithm in computerized tomography; see, e.g., Natterer [14, Chap. V]. In what follows let $f$ be a density distribution compactly supported in $\Omega$. If we assume to know the discrete Radon data $g_{k, j}:=\mathbf{R} f\left(s_{k}, \vartheta_{j}\right)$ for $s_{k}=k / q, k=-q, \ldots, q$, and $\vartheta_{j}=j \pi / p$, $j=0, \ldots, p-1$, then the FBA reconstructs $f_{\mathrm{FB}}$ by

$$
f_{\mathrm{FB}}(x):=\mathbf{R}_{p}^{*} \mathrm{I}_{h}\left(w \star_{q} g\right)(x) .
$$

In the FBA, first the discrete convolution

$$
\left(w \star_{q} g\right)_{\ell, j}:=\frac{1}{q} \sum_{k \in \mathbb{Z}} w_{\ell-k} g_{k, j} \approx\left(v_{\gamma} \star_{s} g\left(\cdot, \vartheta_{j}\right)\right)\left(s_{\ell}\right)
$$

is performed, where $\left\{w_{k}\right\}$ is a weight sequence associated with the chosen kernel $v_{\gamma}$. In the second step, an interpolation operator $\mathrm{I}_{h}$ is applied (with respect to $\ell$ ). Finally, the discrete backprojection operator

$$
\mathbf{R}_{p}^{*} \Phi(x):=\frac{\pi}{p} \sum_{j=0}^{p-1} \Phi\left(x^{t} \omega\left(\vartheta_{j}\right), \vartheta_{j}\right)
$$

is evaluated.

Except for the interpolation process, the discrete convolution (1.5) is the most delicate step in the FBA: the discrete convolution kernel $\left\{w_{k}\right\}$ has to be chosen carefully from the continuous kernel $v_{\gamma}$. For instance, a common choice is

$$
w_{k}=v_{\gamma}\left(s_{k}\right) \text {. }
$$

Here $\gamma$ has to be adjusted to the discretization step size $h=1 / q$. The sensitivity of the reconstructed image to $\gamma$ has been noticed probably for the first time by Smith in $[22$, p. 20]. Rules for selecting $\gamma$ have been suggested by Smith and Keinert [23, Sect. VI], Natterer [14], and Rieder [16]. For local tomography, see Faridani [8] and Rieder, Dietz, and Schuster [17].

Smith [22, pp. 18-19] propagated a different way to define the $w_{k}$ 's. He intended the discrete convolution (1.5) to be exact for a large class of functions. Let $E_{h} u$ be an approximation to the function $u$ given as the superposition of translated and scaled versions of a function $B$; that is,

$$
E_{h} u(s)=\sum_{k \in \mathbb{Z}} u\left(s_{k}\right) B_{h}\left(s-s_{k}\right), \quad \text { where } B_{h}(s)=B(s / h) .
$$


For instance, $E_{h}$ could be an interpolation operator. Defining

$$
w_{k}:=\frac{1}{h} \int v_{\gamma}(s) B_{h}\left(s_{k}-s\right) \mathrm{d} s=\frac{1}{h} v_{\gamma} \star B_{h}\left(s_{k}\right), \quad k \in \mathbb{Z},
$$

we have that

$$
\left(w \star_{q} u\right)_{\ell}=v_{\gamma} \star_{s} E_{h} u\left(s_{\ell}\right), \quad \ell \in \mathbb{Z} .
$$

Moreover, if $E_{h}$ is interpolating, then

$$
\left(w \star_{q} E_{h} u\right)_{\ell}=v_{\gamma} \star_{s} E_{h} u\left(s_{\ell}\right), \quad \ell \in \mathbb{Z}
$$

that is, the discrete convolution (1.5) is exact for $E_{h} u$. Numerical as well as theoretical considerations (see $[16,22]$ ) showed that the reconstructed images $f_{\mathrm{FB}}$ are less sensitive to changes in $\gamma$ when working with (1.9) rather than working with (1.7). Indeed, we will show in the next section that the discrete filter $\left\{w_{k}\right\}$ from (1.9) converges for $\gamma \rightarrow 0$ and that its limit $\left\{w_{k}^{\infty}\right\}$ is again a reconstruction filter belonging to a compactly supported mollifier. This limit filter has an interesting feature: computing $\Lambda E_{h} u\left(s_{\ell}\right) /(2 \pi)$ can now be realized by the discrete convolution

$$
\frac{1}{2 \pi} \Lambda E_{h} u\left(s_{\ell}\right)=\left(w^{\infty} \star_{q} u\right)_{\ell} .
$$

The latter equation is the starting point in section 3 for a reformulation of the FBA leading to optimal $L^{2}$-convergence rates in a semidiscrete setting (Theorem 3.7) where in (1.4) the discrete backprojection operator $\mathbf{R}_{p}^{*}$ is replaced by the continuous one $\mathbf{R}^{*}$. We see how the reconstruction filter, the interpolation process $\left(\mathrm{I}_{h}\right.$ in (1.4)), and the Sobolev regularity of the density distribution $f$ influence the convergence rate. As a by-product of our analysis we discover a new reconstruction filter (Example 4.1) with an improved convergence behavior compared to the widely used Shepp-Logan filter [21] (sections 4 and 5). Indeed, our modified Shepp-Logan filter yields optimal convergence for Sobolev orders up to $5 / 2$, whereas the convergence order of the original Shepp-Logan filter saturates at 2 (Example 5.1). Numerical experiments in the fully discrete setting of (1.4) agree completely with our theoretical predictions and are presented in section 6. Auxiliary but new approximation properties of (quasi-) interpolation operators, which we need for the analysis, are proved in several appendices.

2. The limit. We will now investigate the convergence in Sobolev spaces of $v_{\gamma} \star B$ as $\gamma$ tends to zero. We define the Sobolev spaces $H^{\alpha}\left(\mathbb{R}^{d}\right), \alpha \in \mathbb{R}$, to be the closure of $L^{2}\left(\mathbb{R}^{d}\right)$ with respect to the norm

$$
\|f\|_{\alpha}^{2}:=\int_{\mathbb{R}^{d}}\left(1+\|\xi\|^{2}\right)^{\alpha}|\widehat{f}(\xi)|^{2} \mathrm{~d} \xi
$$

where $\widehat{f}(\xi):=(2 \pi)^{-d / 2} \int_{\mathbb{R}^{d}} f(x) \mathrm{e}^{-\imath \xi^{t} x} \mathrm{~d} x$ is the Fourier transform of a function $f$ in $L^{1}\left(\mathbb{R}^{d}\right) \cap L^{2}\left(\mathbb{R}^{d}\right)$. The Fourier transform can be extended to $L^{2}$-functions and tempered distributions by continuity and duality, respectively. The $\Lambda$-operator,

$$
\widehat{\Lambda f}(\xi):=\|\xi\| \widehat{f}(\xi),
$$

maps $H^{\alpha}\left(\mathbb{R}^{d}\right)$ boundedly to $H^{\alpha-1}\left(\mathbb{R}^{d}\right)$. 
The latter mapping property of $\Lambda$ together with a smoothing effect of $\mathbf{R}$ (see [14, Chap. II, Thm. 5.1]) and the Sobolev embedding theorem shows that $v$ from (1.3) is continuous whenever $e$ is a radially symmetric compactly supported mollifier in $H^{\alpha}\left(\mathbb{R}^{2}\right), \alpha>1$. Furthermore, $v \in L^{1}(\mathbb{R})$; see [16, Lem. 3.1]. Thus, $v_{\gamma} \star B$ is well defined in $H^{t}(\mathbb{R})$ for $B \in H^{t}(\mathbb{R}), t \in \mathbb{R}$; see, e.g., Aubin [1, Prop. 9.3.2].

Lemma 2.1. Let $e \in H^{\alpha}\left(\mathbb{R}^{2}\right), \alpha>1$, be a radially symmetric compactly supported mollifier, and let $v$ be the corresponding reconstruction kernel (1.3). Then,

$$
\lim _{\gamma \rightarrow 0}\left\|v_{\gamma}-\frac{1}{2 \pi} \Lambda \delta\right\|_{-\beta}=0 \quad \text { for any } \beta>3 / 2,
$$

where $\delta$ denotes the Dirac generalized function. Moreover, if $B \in H^{t}(\mathbb{R}), t \in \mathbb{R}$, then

$$
\lim _{\gamma \rightarrow 0}\left\|v_{\gamma} \star B-\frac{1}{2 \pi} \Lambda B\right\|_{t-1}=0 .
$$

For values of $s$ such that $\Lambda B$ is continuous near $s$ we have

$$
\lim _{\gamma \rightarrow 0} v_{\gamma} \star B(s)=\frac{1}{2 \pi} \Lambda B(s) .
$$

Proof. We prove (2.2) which then implies (2.1) when setting $B=\delta$ and recalling that $\delta \in H^{t}(\mathbb{R})$ for $t<-1 / 2$. With

$$
I(\xi, \gamma)=\left(1+|\xi|^{2}\right)^{t-1}\left|\sqrt{2 \pi} \widehat{v_{\gamma}}(\xi) \widehat{B}(\xi)-\right| \xi|\widehat{B}(\xi) /(2 \pi)|^{2}
$$

we obtain that

$$
\left\|v_{\gamma} \star B-\frac{1}{2 \pi} \Lambda B\right\|_{t-1}^{2}=\int_{\mathbb{R}} I(\xi, \gamma) \mathrm{d} \xi .
$$

By the projection slice theorem (see, e.g., Natterer [14, Chap. II, Thm. 1.1]) we find ( $e$ is a radially symmetric function)

$$
\widehat{v_{\gamma}}(\xi)=\frac{1}{2 \pi}|\xi| \widehat{\mathbf{R} e_{\gamma}}(\xi)=\frac{1}{\sqrt{2 \pi}}|\xi| \widehat{e_{\gamma}}(\xi, 0)=\frac{1}{\sqrt{2 \pi}}|\xi| \widehat{e}(\gamma \xi, 0),
$$

which yields

$$
I(\xi, \gamma) \leq\left(1+|\xi|^{2}\right)^{t}|\widehat{B}(\xi)|^{2}|\widehat{e}(\gamma \xi, 0)-1 /(2 \pi)|^{2} .
$$

The stated convergence follows now from $\widehat{e}(0,0)=1 /(2 \pi)$, the Riemann-Lebesgue lemma, and the dominated convergence theorem.

Let us look at an example. For $\chi$ being the indicator function of the interval $[-1 / 2,1 / 2]$ we are able to compute $\Lambda \chi$ by

$$
\Lambda \chi(s)=-\frac{1}{\pi} \int_{\mathbb{R}}|s-t|^{-2} \chi(t) \mathrm{d} t, \quad|s|>1 / 2 ;
$$

see Faridani et al. [9, Form. (2.1)]. Evaluating the integral gives

$$
\Lambda \chi(s)=\frac{4}{\pi} \frac{1}{1-4 s^{2}}
$$


The above formula holds for all $s \in \mathbb{R} \backslash\{-1 / 2,1 / 2\}$. This can be verified using the relation $\Lambda(1-\chi)=-\Lambda \chi$ and applying formula (2.1) of [9] to $1-\chi$, the indicator function of $\mathbb{R} \backslash[-1 / 2,1 / 2]$. So we have that

$$
\lim _{\gamma \rightarrow 0} v_{\gamma} \star \chi(s)=\frac{2}{\pi^{2}} \frac{1}{1-4 s^{2}}, \quad|s| \neq 1 / 2 .
$$

In weaker Sobolev norms we can even give convergence rates. For formulating the respective result and later in the paper we use the following convenient notation: $A \lesssim B$ indicates the existence of a generic constant $c$ such that $A \leq c B$ holds uniformly with respect to all parameters $A$ and $B$ may depend on.

Corollary 2.2. Let $0 \leq s \leq 2$. Under the assumptions of Lemma 2.1 we have that

$$
\left\|v_{\gamma} \star B-\frac{1}{2 \pi} \Lambda B\right\|_{t-1-s} \lesssim \gamma^{s}\|B\|_{t} .
$$

Proof. As in the proof of Lemma 2.1 we obtain that

$$
\left\|v_{\gamma} \star B-\frac{1}{2 \pi} \Lambda B\right\|_{t-1-s}^{2} \leq \int_{\mathbb{R}}\left(1+|\xi|^{2}\right)^{t-s}|\widehat{B}(\xi)|^{2} M(\gamma \xi, 0) \mathrm{d} \xi,
$$

where $M(z):=|\widehat{e}(z)-1 /(2 \pi)|^{2}, z \in \mathbb{R}^{2}$. Since $e$ is an even function all its first order moments vanish. Therefore, all first order derivatives of $\widehat{e}$ are zero at the origin. Thus the Taylor expansion of $\widehat{e}$ about the origin becomes

$$
\widehat{e}(z)=\frac{1}{2 \pi}+\sum_{\substack{\nu \in \mathbb{N}_{0}^{2} \\ \nu_{1}+\nu_{2}=2}} \frac{D^{\nu} \widehat{e}\left(\tau_{z} z\right)}{\nu !} z^{\nu} \quad \text { for a } \tau_{z} \in[0,1],
$$

which yields $M(z) \lesssim\|z\|^{4}$. Now let $s \in[0,2]$. Then,

$$
\begin{aligned}
\left\|v_{\gamma} \star B-\frac{1}{2 \pi} \Lambda B\right\|_{t-1-s}^{2} \lesssim & \int_{|\xi| \leq 1 / \gamma}|\widehat{B}(\xi)|^{2}\left(1+|\xi|^{2}\right)^{t-s} M(\gamma \xi, 0) \mathrm{d} \xi \\
& +\int_{|\xi|>1 / \gamma}|\widehat{B}(\xi)|^{2}\left(1+|\xi|^{2}\right)^{t-s} \mathrm{~d} \xi \\
\lesssim & \int_{|\xi| \leq 1 / \gamma}|\widehat{B}(\xi)|^{2}\left(1+|\xi|^{2}\right)^{t}|\xi|^{4-2 s} \mathrm{~d} \xi \\
& +\int_{|\xi|>1 / \gamma}|\widehat{B}(\xi)|^{2}\left(1+|\xi|^{2}\right)^{t}|\xi|^{-2 s} \mathrm{~d} \xi .
\end{aligned}
$$

Both latter terms can be bounded by $\gamma^{2 s}\|B\|_{t}^{2}$.

Remark 2.3. The generalization of Corollary 2.2 to reconstruction kernels $v$ belonging to mollifiers with higher order vanishing moments is obvious.

3. The FBA is optimal. We will reformulate the FBA (1.4) for the limit filters considered in the former section; see (3.2) below. This new representation of the FBA allows us to introduce a novel error analysis which shows that the FBA is optimal for tomographic inversion. 
3.1. A new representation of the FBA. We start with the following simple observation.

Lemma 3.1. Let $B$ be in $H^{t}(\mathbb{R})$ for a $t \in \mathbb{R}$ such that $\Lambda B(s)$ is continuous near integer values of $s$. For $\psi(s)=\sum_{k \in \mathbb{Z}} c_{k} B_{h}(s-h k)$, where $\left\{c_{k}\right\}$ is a finite sequence and $h$ is positive, we have

$$
\Lambda \psi(h \ell)=h^{-1} \sum_{k \in \mathbb{Z}} c_{k} \Lambda B(\ell-k), \quad \ell \in \mathbb{Z} .
$$

Proof. The statement follows directly from the relations $\Lambda B_{h}(s)=\Lambda B(s / h) / h$ and $\Lambda T^{a}=T^{a} \Lambda$, where $T^{a}$ is the translation operator $T^{a} u(s)=u(s-a)$.

Remark 3.2. Relying on Lemma 3.1 we easily derive that

$$
\frac{2}{\pi} \frac{1}{q+1 / 2}=\sum_{k=-q}^{q} \Lambda \chi(k) \text { for any } q \in \mathbb{N}_{0},
$$

where $\chi$ is as in (2.3). To prove the above identity we mention only that $\sum_{k=-q}^{q} \chi(\cdot-$ $k)$ is the characteristic function of the interval $[-q-1 / 2, q+1 / 2]$.

Let the operator $E_{h}$ be given by (1.8) with $B$ as in Lemma 3.1. Define the discrete reconstruction kernel $\left\{w_{k}^{\infty}\right\}$ by

$$
w_{k}^{\infty}=\frac{1}{2 \pi h^{2}} \Lambda B(k)=v_{h}^{\infty}(h k)
$$

where $v_{h}^{\infty}(s)=v^{\infty}(s / h) / h^{2}$ and $v^{\infty}(s):=\Lambda B(s) /(2 \pi)$. Then, the discrete convolution (1.5) can be written as the $\Lambda$-operator applied exactly to a function approximating $g$ from discrete values:

$$
\left(w^{\infty} \star_{q} g\right)_{\ell, j}=\frac{1}{2 \pi}\left(\Lambda E_{h} g\left(\cdot, \vartheta_{j}\right)\right)(h \ell), \quad \ell \in \mathbb{Z} .
$$

Thus, the reconstructed image $f_{\mathrm{FB}}$ may be rewritten as

$$
f_{\mathrm{FB}}(x)=\frac{1}{2 \pi} \mathbf{R}_{p}^{*} \mathrm{I}_{h} \Lambda E_{h} g(x) ;
$$

see (1.4). Please observe that the three operators $E_{h}, \Lambda$, and $\mathrm{I}_{h}$ act on the first variable of the data $g=\mathbf{R} f$.

Example 3.3. Let $B=\chi$ be the characteristic function of $[-1 / 2,1 / 2]$. Then, the reconstruction kernel $w^{\infty}$ used for evaluating (3.2) is

$$
w_{k}^{\infty}=\frac{2}{\pi^{2} h^{2}} \frac{1}{1-4 k^{2}},
$$

which follows from (2.4) and (3.1). Here, $w^{\infty}$ is the discrete Shepp-Logan reconstruction filter [21].

Remark 3.4. Let the discrete reconstruction kernel $\left\{w_{k}\right\}$ be given by (1.9). Due to Lemma 2.1 we obtain $\lim _{\gamma \rightarrow 0} w_{k}=w_{k}^{\infty}$, implying that

$$
\lim _{\gamma \rightarrow 0}\left(w \star_{q} g\right)_{\ell, j}=\frac{1}{2 \pi}\left(\Lambda E_{h} g\left(\cdot, \vartheta_{j}\right)\right)(h \ell), \quad \ell \in \mathbb{Z} .
$$

We next ask the question, Which mollifier $e^{\infty}$ belongs to the reconstruction kernel $v^{\infty}(3.1)$ ? By (1.3) and the projection slice theorem we find that

$$
\widehat{e^{\infty}}(\xi)=\widehat{B}(\|\xi\|) / \sqrt{2 \pi}, \quad \xi \in \mathbb{R}^{2}
$$



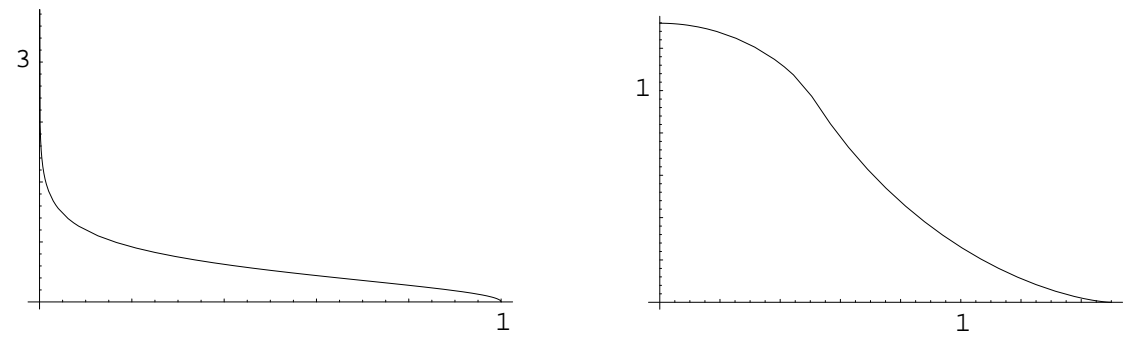

FIG. 1. Radial part of limit mollifier $e^{\infty}$ (3.4) where $B$ is the linear (left) and the quadratic (right) B-spline, respectively.

which yields ( $\mathrm{J}_{0}$ denoting the Bessel function of the first kind of order 0$)$

$$
e^{\infty}(x)=\frac{1}{\sqrt{2 \pi}} \int_{0}^{\infty} r \widehat{B}(r) \mathrm{J}_{0}(\|x\| r) \mathrm{d} r .
$$

In view of (3.3) the mollifier $e^{\infty}$ is in $L^{2}\left(\mathbb{R}^{2}\right)$ if $\lim _{r \rightarrow \infty} r|\widehat{B}(r)|=0$. Further, a compact support of $B$ implies a compact support of $e^{\infty}$. More precisely, let $B$ be even with supp $B \subset[-R, R]$; then $\operatorname{supp} e^{\infty} \subset\left\{x \in \mathbb{R}^{2} \mid\|x\| \leq R\right\}$. The latter statement is a consequence from the Paley-Wiener theorems; see, e.g., Rudin [19, Chap. 7].

Example 3.5. Let $B=\chi$ be the characteristic function of the interval $[-1 / 2,1 / 2]$. By formula 6.671.7 from [11] we find that

$$
e^{\infty}(x)=\left\{\begin{array}{ccc}
\frac{2}{\pi} \frac{1}{\sqrt{1-4\|x\|^{2}}} & : & \|x\|<1 / 2 \\
0 & : & \text { otherwise }
\end{array}\right.
$$

which is the mollifier belonging to $v^{\infty}(s)=\frac{1}{2 \pi} \Lambda \chi(s)=\frac{2}{\pi^{2}}\left(1-4 s^{2}\right)^{-1}$; see (2.4). The graphs of the radial parts of $e^{\infty}$ with respect to the linear and quadratic B-splines are plotted in Figure 1.

3.2. A novel error estimate. The new representation (3.2) of the FBA gives us the freedom to provide a novel error analysis based on principles from approximation theory. Indeed, we will be able to prove $L^{2}$-convergence of the FBA with optimal rates.

In contrast, the error estimates based on Fourier analysis (see Natterer [14, Chap. V] and Faridani and Ritman [10]) are of qualitative nature in terms of essentially band-limited functions. Since the main tool used is the Poisson summation formula the considered density distributions are required to be continuous functions at least $\left(\widehat{f} \in L^{1}\right)$. Convergence has been shown before: Popov [15] established pointwise convergence restricted to a small class of functions (piecewise $\mathcal{C}^{\infty}$ with jumps across smooth curves). The approach of Rieder and Schuster [18] leads to $L^{2}$-convergence for $f \in H_{0}^{\alpha}(\Omega), \alpha>1 / 2$, however, with suboptimal rates.

In our analysis below we will not take into account the error introduced by discretizing the backprojection $\mathbf{R}^{*}$; that is, our model of the FBA reconstructs $\widetilde{f}_{\mathrm{FB}}$ by

$$
\widetilde{f}_{\mathrm{FB}}(x):=\frac{1}{2 \pi} \mathbf{R}^{*} \mathrm{I}_{h} \Lambda E_{h} \mathbf{R} f(x)
$$

compare (3.2). 
Before bounding the reconstruction error of $\widetilde{f}_{\mathrm{FB}}$ we generalize both operators $E_{h}$ and $\mathrm{I}_{h}$. For $u \in H^{\alpha}(\mathbb{R}), \alpha \in \mathbb{R}$, we define

$$
E_{h} u(s):=h^{-1} \sum_{k \in \mathbb{Z}}\left\langle u, \epsilon_{h}\left(\cdot-s_{k}\right)\right\rangle B_{h}\left(s-s_{k}\right),
$$

where $\epsilon_{h}(s)=\epsilon(s / h)$ with $\epsilon \in H^{-\alpha}(\mathbb{R})$ being even and $\widehat{\epsilon}(0)=1 / \sqrt{2 \pi}$. Further, $\langle\cdot, \cdot\rangle$ denotes the duality pairing in $H^{\alpha}(\mathbb{R}) \times H^{-\alpha}(\mathbb{R})$. For $u \in H^{\alpha}(\mathbb{R}), \alpha>1 / 2$, we may choose $\epsilon=\delta$ (Dirac distribution). Thus, $h^{-1}\left\langle u, \epsilon_{h}\left(\cdot-s_{k}\right)\right\rangle=u\left(s_{k}\right)$, and the general form (3.6) of $E_{h}$ coincides with its former definition (1.8). We extended the domain of definition of $E_{h}$ to cover (generalized) functions in $H^{\alpha}(\mathbb{R})$ with $\alpha \leq 1 / 2$.

The redefinition of $E_{h}$ was necessary because we apply $E_{h}$ to $\mathbf{R} f(\cdot, \vartheta)$ (see (3.5)), and we have only that $\mathbf{R} f(\cdot, \vartheta) \in H_{0}^{1 / 2}(-1,1)$ for $f \in L^{2}(\Omega)$ and almost all $\vartheta$. Moreover, our new model allows for finite width of the rays and detector inhomogeneities in the observed semidiscrete Radon data; see Natterer [14, Chap. V.5.1]. Indeed, for $\epsilon$ being a nonnegative function compactly supported in $[-1 / 2,1 / 2]$ with a normalized mean value we obtain

$$
h^{-1}\left\langle\mathbf{R} f(\cdot, \vartheta), \epsilon_{h}\left(\cdot-s_{k}\right)\right\rangle=h^{-1} \int_{s_{k}-h / 2}^{s_{k}+h / 2} \mathbf{R} f(s, \vartheta) \epsilon_{h}\left(s-s_{k}\right) \mathrm{d} s .
$$

Hence, $\epsilon$ can be seen as the sensitivity profile of the X-ray detectors.

In a very similar way we define $\mathrm{I}_{h}$ by

$$
\mathrm{I}_{h} u(s):=h^{-1} \sum_{k \in \mathbb{Z}}\left\langle u, \eta_{h}\left(\cdot-s_{k}\right)\right\rangle A_{h}\left(s-s_{k}\right),
$$

where $\eta$ and $A$ are like $\epsilon$ and $B$ from (3.6), respectively.

Our modifications of $E_{h}$ and $\mathrm{I}_{h}$ have no effect on the efficient computation of $\mathrm{I}_{h} \Lambda E_{h} \mathbf{R} f(\cdot, \vartheta) /(2 \pi)$ by discrete convolution. A straightforward calculation reveals that

$$
\frac{1}{2 \pi}\left(\mathrm{I}_{h} \Lambda E_{h} \mathbf{R} f(\cdot, \vartheta)\right)(s)=\sum_{\ell \in \mathbb{Z}}\left(w \star_{q} g^{\epsilon}(\cdot, \vartheta)\right)_{\ell} A_{h}\left(s-s_{\ell}\right),
$$

where $g^{\epsilon}(s, \vartheta)=\left(\mathbf{R} f(\cdot, \vartheta) \star_{s} \epsilon_{h}\right)(s) / h$ (see (3.7)), and the discrete reconstruction kernel $w$ is given by

$$
\begin{aligned}
& w_{r}=v(r) / h^{2}, \quad r \in \mathbb{Z}, \\
& \text { with } v(s):=\frac{1}{\pi} \int_{0}^{\infty} \sigma \widehat{B}(\sigma) \widehat{\eta}(\sigma) \cos (s \sigma) \mathrm{d} \sigma .
\end{aligned}
$$

The above integral exists as a duality pairing whenever $B \in H^{t}(\mathbb{R})$ and $\eta \in H^{1-t}(\mathbb{R})$.

Example 3.6. We will give the Shepp-Logan reconstruction filter a new interpretation. To this end, let $B(s)=\operatorname{sinc}(\pi s)$ be the interpolating function used to define $E_{h}$. In $\mathrm{I}_{h}$ let $\eta$ be the characteristic function of the interval $[-1 / 2,1 / 2]$. We obtain $\widehat{B}=$ $\chi_{[-\pi, \pi]} / \sqrt{2 \pi}\left(\chi_{D}\right.$ characteristic function of interval $\left.D\right)$ and $\widehat{\eta}(\sigma)=\operatorname{sinc}(\sigma / 2) / \sqrt{2 \pi}$. Hence,

$$
v(s)=\frac{2}{\pi^{2}} \frac{2 s \sin (\pi s)-1}{4 s^{2}-1} \quad \text { and } \quad w_{k}=v(k) / h^{2}=\frac{2}{\pi^{2} h^{2}} \frac{1}{1-4 k^{2}} ;
$$


see Example 3.3 and compare formula (1.22) on page 111 in [14].

In estimating the reconstruction error below we will need that the inversion formula (1.1) holds true for functions in $L^{2}(\Omega)$; that is,

$$
f=(2 \pi)^{-1} \mathbf{R}^{*} \Lambda \mathbf{R} f \quad \text { for any } f \in L^{2}(\Omega) .
$$

As far as we know, the most general version of (1.1) is due to Smith, Solomon, and Wagner [24, p. 1257] requiring a compactly supported $f \in H^{\alpha}\left(\mathbb{R}^{2}\right)$ with $\alpha \geq 1 / 2$. To verify (3.10) we recall the following mapping property of the Radon transform:

$$
\mathbf{R}: H_{0}^{\alpha}(\Omega) \rightarrow H^{(\alpha+1 / 2,0)} \quad \text { is bounded for any } \alpha \geq 0,
$$

which is due to Louis and Natterer [13, Thm. 3.1]; see also [14, Thm. II.5.1]. Above, $H_{0}^{\alpha}(\Omega)$ is the closure of $\mathcal{C}_{0}^{\infty}(\Omega)$, the space of infinitely differentiable functions compactly supported in $\Omega$, with respect to the norm $\|\cdot\|_{\alpha}$. Further, $H^{(\beta, 0)}$ is the tensor product space $H^{\beta}(\mathbb{R}) \widehat{\otimes} L^{2}(0, \pi)$.

Now the validity of (3.10) can be seen from the following three facts: 1 . The operator $\mathbf{R}^{*} \Lambda \mathbf{R}: L^{2}(\Omega) \rightarrow L^{2}(\Omega)$ is bounded since all three mappings $\mathbf{R}: L^{2}(\Omega) \rightarrow$ $H^{(1 / 2,0)}, \Lambda: H^{(1 / 2,0)} \rightarrow H^{(-1 / 2,0)}$, and $\mathbf{R}^{*}: H^{(-1 / 2,0)} \rightarrow L^{2}(\Omega)$ are bounded. ${ }^{1}$ 2. Formula (3.10) applies to all $f \in \mathfrak{C}_{0}^{\infty}(\Omega)$; see, e.g., Natterer [14, Thm. II.2.1]. 3 . The space $\mathcal{C}_{0}^{\infty}(\Omega)$ is dense in $L^{2}(\Omega)$.

After these preparations we concentrate on the reconstruction error for $f$ in $H_{0}^{\alpha}(\Omega), \alpha \geq 0$. Relying on (3.10) we begin with

$$
\begin{aligned}
\left\|\tilde{f}_{\mathrm{FB}}-f\right\|_{L^{2}(\Omega)} & =\frac{1}{2 \pi}\left\|\mathbf{R}^{*} I_{h} \Lambda E_{h} \mathbf{R} f-\mathbf{R}^{*} \Lambda \mathbf{R} f\right\|_{L^{2}(\Omega)} \\
\leq\left\|\left(\mathbf{R}^{*} I_{h}-\mathbf{R}^{*}\right) \Lambda E_{h} \mathbf{R} f\right\|_{L^{2}(\Omega)} & \\
& +\left\|\mathbf{R}^{*} \Lambda\left(E_{h} \mathbf{R} f-\mathbf{R} f\right)\right\|_{L^{2}(\Omega)}
\end{aligned}
$$

and proceed by estimating both norms on the right-hand side.

We saw above that $\mathbf{R}^{*} \Lambda$ maps $H^{(1 / 2,0)}$ boundedly to $L^{2}(\Omega)$. Hence,

$$
\left\|\mathbf{R}^{*} \Lambda\left(E_{h} \mathbf{R} f-\mathbf{R} f\right)\right\|_{L^{2}(\Omega)} \lesssim\left\|E_{h} \mathbf{R} f-\mathbf{R} f\right\|_{H^{(1 / 2,0)}} .
$$

Now we need an approximation property of $E_{h}$. Therefore, we assume there are nonnegative constants $\tau_{\max }$ and $\beta_{\min } \leq \beta_{\max }$ such that

$$
\begin{gathered}
\left\|E_{h} u-u\right\|_{\tau} \lesssim h^{\beta-\tau}\|u\|_{\beta} \quad \text { as } h \rightarrow 0 \\
\text { for } \beta_{\min } \leq \beta \leq \beta_{\max }, 0 \leq \tau \leq \beta, \quad \tau \leq \tau_{\max }, u \in H_{0}^{\beta}(-1,1) .
\end{gathered}
$$

For instance, if $E_{h}$ represents piecewise linear interpolation ${ }^{2}$, then (3.12) holds with $\beta_{\max }=2, \beta_{\min }>1 / 2$, and $\tau_{\max }<3 / 2$. For piecewise linear interpolation the approximation property (3.12) is a classical result when $\tau \in\{0,1\}$ and $\beta=2$; see, e.g., Strang and Fix [25, Thm. 1.3]. Also band-limited interpolation ${ }^{3}$ yields (3.12) with $\beta_{\min }>1 / 2$ and any $\beta_{\max }=\tau_{\max }<\infty$. In Appendices $\mathrm{A}$ and $\mathrm{B}$ we prove (3.12) for more general interpolation-like operators $E_{h}$ where $\beta_{\min }=0$.

\footnotetext{
${ }^{1}$ The continuity of $\mathbf{R}^{*}: H^{(-1 / 2,0)} \rightarrow L^{2}(\Omega)$ follows from (3.11) by duality.

${ }^{2} \epsilon$ is the Dirac distribution and $B$ is the linear B-spline.

${ }^{3} \epsilon$ is the Dirac distribution and $B(x)=\operatorname{sinc}(\pi x)$.
} 
Estimates of terms from above by powers of $h$ (like (3.12)) are in what follows always understood asymptotically in the sense of $h \rightarrow 0$.

Assume (3.12) to hold with $\beta_{\max } \geq 1 / 2$ and $\tau_{\max } \geq 1 / 2$. If $\max \left\{0, \beta_{\min }-1 / 2\right\} \leq$ $\alpha \leq \beta_{\max }-1 / 2$, then

$$
\left\|\mathbf{R}^{*} \Lambda\left(E_{h} \mathbf{R} f-\mathbf{R} f\right)\right\|_{L^{2}(\Omega)} \lesssim h^{\alpha}\|\mathbf{R} f\|_{H^{(1 / 2+\alpha, 0)}} \stackrel{(3.11)}{\lesssim} h^{\alpha}\|f\|_{\alpha}
$$

Now we turn to $\left\|\left(\mathbf{R}^{*} \mathrm{I}_{h}-\mathbf{R}^{*}\right) \Lambda E_{h} \mathbf{R} f\right\|_{L^{2}(\Omega)}$ which we estimate according to

$$
\begin{aligned}
& \left\|\mathbf{R}^{*}\left(\mathrm{I}_{h}-I\right) \Lambda E_{h} \mathbf{R} f\right\|_{L^{2}(\Omega)} \\
& \leq\left\|\mathbf{R}^{*}\right\|_{H^{(-1 / 2,0)} \rightarrow L^{2}(\Omega)}\left\|\mathrm{I}_{h}-I\right\|_{H^{\alpha-1 / 2}(\mathbb{R}) \rightarrow H^{-1 / 2}(\mathbb{R})} \\
& \quad \times\|\Lambda\|_{H^{\alpha+1 / 2}(\mathbb{R}) \rightarrow H^{\alpha-1 / 2}(\mathbb{R})}\left\|E_{h} \mathbf{R} f\right\|_{H^{(1 / 2+\alpha, 0)}},
\end{aligned}
$$

where $I: H^{\alpha-1 / 2}(\mathbb{R}) \hookrightarrow H^{-1 / 2}(\mathbb{R})$ is the canonical inclusion. Observe that (3.12) implies the boundedness of $E_{h}: H_{0}^{1 / 2+\alpha}(-1,1) \rightarrow H^{1 / 2+\alpha}(\mathbb{R})$ uniformly in $h$ for $0 \leq \alpha \leq \min \left\{\beta_{\max }, \tau_{\max }\right\}-1 / 2$. Thus,

$$
\left\|E_{h} \mathbf{R} f\right\|_{H^{(1 / 2+\alpha, 0)}} \lesssim\|\mathbf{R} f\|_{H^{(1 / 2+\alpha, 0)}} \stackrel{(3.11)}{\lesssim}\|f\|_{\alpha} .
$$

For the operator $\mathrm{I}_{h}$ we require that

$$
\left\|\mathrm{I}_{h}-I\right\|_{H^{\alpha-1 / 2}(\mathbb{R}) \rightarrow H^{-1 / 2}(\mathbb{R})} \lesssim h^{\alpha} \quad \text { as } h \rightarrow 0 \text { for } 0 \leq \alpha \leq \alpha_{\mathrm{I}}
$$

which yields that

$$
\left\|\left(\mathbf{R}^{*} \mathrm{I}_{h}-\mathbf{R}^{*}\right) \Lambda E_{h} \mathbf{R} f\right\|_{L^{2}(\Omega)} \lesssim h^{\alpha}\|f\|_{\alpha}
$$

Thus, we have proven the following theorem.

TheOREM 3.7. Assume (3.12) to hold with $\beta_{\max } \geq 1 / 2$ and $\tau_{\max } \geq 1 / 2$. Further, let there exist an $\alpha_{\mathrm{I}}>0$ such that (3.13) holds true. then

$$
\text { If } \max \left\{0, \beta_{\min }-1 / 2\right\} \leq \alpha \leq \min \left\{\alpha_{\mathrm{I}}, \beta_{\max }-1 / 2, \tau_{\max }-1 / 2\right\} \text { and } f \in H_{0}^{\alpha}(\Omega) \text {, }
$$

$$
\left\|f-\frac{1}{2 \pi} \mathbf{R}^{*} \mathrm{I}_{h} \Lambda E_{h} \mathbf{R} f\right\|_{L^{2}(\Omega)} \lesssim h^{\alpha}\|f\|_{\alpha} \quad \text { as } h \rightarrow 0 .
$$

The best possible $L^{2}$-convergence rate for the reconstruction of $f \in H_{0}^{\alpha}(\Omega)$ from Radon data sampled at distance $h$ is $h^{\alpha}$ as $h \rightarrow 0$; see Natterer [14, Chap. IV, Thm. 2.2]. So we just proved that the FBA with an "averaged" limit kernel (3.9) is an optimal reconstruction algorithm (at least for semidiscrete data). The range of Sobolev orders yielding optimal convergence depends on the chosen filter and the used interpolation procedure.

Theorem 3.7 looks similar to Theorem V.1.2 of Natterer [14]. The main difference is that our theorem takes the discretization of the convolution into account. On the other hand, the main result of Popov [15, Thm. 3, p. 35] investigates pointwise convergence utilizing an approach based on asymptotic expansions. It does consider the fully discrete algorithm but is applicable to a smaller class of functions (piecewise $\mathrm{e}^{\infty}$ with jumps across smooth curves), is stated without a detailed proof, and is not always easily applied to concrete examples. 
Example 3.8. Here we provide a simple example for (3.13) which results in a convergence proof of the FBA with the Shepp-Logan filter and nearest-neighbor interpolation.

To this end let both $\eta$ and $A$ be first order B-splines; that is, $\eta=A=\chi_{[-1 / 2,1 / 2[}$. In this situation (3.13) applies with $\alpha_{\mathrm{I}}=3 / 2$ as we will demonstrate now. By Theorem A.2,

$$
\left\|\mathrm{I}_{h} u-u\right\|_{\tau} \lesssim h^{\beta-\tau}\|u\|_{\beta}
$$

for $0 \leq \tau \leq \beta \leq 1$ and $\tau<1 / 2$. To estimate $\left\|\mathrm{I}_{h} u-u\right\|_{-1 / 2}$ we use a duality argument and the symmetry $\mathrm{I}_{h}=\mathrm{I}_{h}^{*}$, where $\mathrm{I}_{h}^{*}$ is the $L^{2}$-adjoint of $\mathrm{I}_{h}$. We find that, for $0 \leq \alpha \leq 1 / 2$,

$$
\begin{gathered}
\left\|\mathrm{I}_{h} u-u\right\|_{-1 / 2}=\sup _{v \in H^{1 / 2}(\mathbb{R})} \frac{\left\langle\mathrm{I}_{h} u-u, v\right\rangle}{\|v\|_{1 / 2}}=\sup _{v \in H^{1 / 2}(\mathbb{R})} \frac{\left\langle u, \mathrm{I}_{h} v-v\right\rangle}{\|v\|_{1 / 2}} \\
\leq\|u\|_{\alpha-1 / 2} \sup _{v \in H^{1 / 2}(\mathbb{R})} \frac{\left\|\mathrm{I}_{h} v-v\right\|_{1 / 2-\alpha}}{\|v\|_{1 / 2}} \stackrel{(3.15)}{\lesssim} h^{\alpha}\|u\|_{\alpha-1 / 2} .
\end{gathered}
$$

For $1 / 2<\alpha \leq 3 / 2$ we estimate similarly, relying on $\mathrm{I}_{h}^{2}=\mathrm{I}_{h}$,

$$
\begin{aligned}
& \left\|\mathrm{I}_{h} u-u\right\|_{-1 / 2}=\sup _{v \in H^{1 / 2}(\mathbb{R})} \frac{\left\langle\left(\mathrm{I}_{h}-I\right)^{2} u, v\right\rangle}{\|v\|_{1 / 2}}=\sup _{v \in H^{1 / 2}(\mathbb{R})} \frac{\left\langle\mathrm{I}_{h} u-u, \mathrm{I}_{h} v-v\right\rangle}{\|v\|_{1 / 2}} \\
& \leq\left\|\mathrm{I}_{h} u-u\right\|_{L^{2}(\mathbb{R})} \sup _{v \in H^{1 / 2}(\mathbb{R})} \frac{\left\|\mathrm{I}_{h} v-v\right\|_{L^{2}(\mathbb{R})}}{\|v\|_{1 / 2}} \\
& \stackrel{(3.15)}{\lesssim} h^{\alpha-1 / 2}\|u\|_{\alpha-1 / 2} h^{1 / 2} .
\end{aligned}
$$

Hence, (3.13) holds for $\alpha_{\mathrm{I}}=3 / 2$.

Recalling Example 3.6 we observe that the FBA with the Shepp-Logan filter and nearest-neighbor interpolation is represented by $B(s)=\operatorname{sinc}(\pi s)$ and $\eta=A=$ $\chi_{[-1 / 2,1 / 2[}$ in our framework (3.5). Therefore, our results from Appendix B give that

$$
\left\|\tilde{f}_{\mathrm{FB}}-f\right\|_{L^{2}(\Omega)} \lesssim h^{\min \{3 / 2, \alpha\}}\|f\|_{\alpha} \quad \text { for } f \in H_{0}^{\alpha}(\Omega), \alpha>0
$$

as long as $\epsilon$ is either an even, compactly supported, and normalized $L^{2}$-function (Theorem B.2) or the Dirac distribution (Theorem B.4).

In the next section we will generalize the above example, covering especially piecewise linear interpolation in $\mathrm{I}_{h}$.

So far we have not shown $L^{2}$-convergence of the FBA when the density distribution $f$ is only in $L^{2}(\Omega)$. However, we possess all the tools to do this.

Corollary 3.9. Assume (3.12) to hold with $\beta_{\min } \leq 1 / 2, \beta_{\max }>1 / 2$, and $\tau_{\max }>1 / 2$. Further, let there exist an $\alpha_{\mathrm{I}}>0$ such that (3.13) holds true. Then,

$$
\lim _{h \rightarrow 0}\left\|f-\frac{1}{2 \pi} \mathbf{R}^{*} I_{h} \Lambda E_{h} \mathbf{R} f\right\|_{L^{2}(\Omega)}=0 \quad \text { for any } f \in L^{2}(\Omega) .
$$

Proof. We will use that $\mathbf{R}^{*} \mathrm{I}_{h} \Lambda E_{h} \mathbf{R}: L^{2}(\Omega) \rightarrow L^{2}(\Omega)$ is uniformly bounded in $h>0$. This follows by setting $\alpha=0$ in (3.14) which is allowed since $\beta_{\min } \leq 1 / 2$. Thus, $\left\|\mathbf{R}^{*} I_{h} \Lambda E_{h} \mathbf{R}\right\|_{L^{2}(\Omega) \rightarrow L^{2}(\Omega)} \lesssim 1$. 
Fix an $\alpha$ with $0<\alpha \leq \min \left\{\alpha_{\mathrm{I}}, \beta_{\max }-1 / 2, \tau_{\max }-1 / 2\right\}$. By assumption the upper bound on $\alpha$ is positive. Since $H_{0}^{\alpha}(\Omega)$ is dense in $L^{2}(\Omega)$ there exists a family $\left\{f_{\lambda}\right\}_{\lambda>0} \subset H_{0}^{\alpha}(\Omega)$ which converges to $f$ in $L^{2}(\Omega)$ as $\lambda \rightarrow 0$. Without loss of generality we may assume that $f$ is not an element of $H_{0}^{\beta}(\Omega)$ for any $\beta>0$ (otherwise we apply Theorem 3.7 to obtain (3.18)). Therefore, the function $\rho(\lambda):=\left\|f_{\lambda}\right\|_{\alpha}$ explodes: $\rho(\lambda) \rightarrow \infty$ as $\lambda \rightarrow 0$. Now we choose a family $\left\{\lambda_{h}\right\}_{h>0}$ satisfying

$$
\lim _{h \rightarrow 0} \lambda_{h}=0 \quad \text { as well as } \quad \lim _{h \rightarrow 0} h^{\alpha} \rho\left(\lambda_{h}\right)=0 .
$$

We proceed with

$$
\begin{aligned}
\left\|f-\frac{1}{2 \pi} \mathbf{R}^{*} \mathrm{I}_{h} \Lambda E_{h} \mathbf{R} f\right\|_{L^{2}(\Omega)} \leq & \left\|f-f_{\lambda_{h}}\right\|_{L^{2}(\Omega)} \\
& +\left\|f_{\lambda_{h}}-\frac{1}{2 \pi} \mathbf{R}^{*} \mathrm{I}_{h} \Lambda E_{h} \mathbf{R} f_{\lambda_{h}}\right\|_{L^{2}(\Omega)} \\
& +\left\|\mathbf{R}^{*} \mathrm{I}_{h} \Lambda E_{h} \mathbf{R}\left(f_{\lambda_{h}}-f\right)\right\|_{L^{2}(\Omega)} \\
& \lesssim\left\|f-f_{\lambda_{h}}\right\|_{L^{2}(\Omega)}+h^{\alpha} \rho\left(\lambda_{h}\right),
\end{aligned}
$$

where we applied Theorem 3.7 in the last step. Finally, the limit $h \rightarrow 0$ implies (3.18).

Example 3.10. We reconsider Example 3.8 in light of Corollary 3.9. The convergence (3.18) holds true when using the Shepp-Logan filter with nearest-neighbor interpolation for $\mathrm{I}_{h}$ and band-limited quasi interpolation for $E_{h}$; that is, $\eta=A=\chi_{[-1 / 2,1 / 2[}, B(s)=\operatorname{sinc}(\pi s)$, and $\epsilon$ is an even, compactly supported, and normalized $L^{2}$-function (Theorem B.2). Please note that band-limited interpolation for $E_{h}$ ( $\epsilon$ is the Dirac distribution), which requires $\beta_{\min }>1 / 2$, is not covered by Corollary 3.9.

4. Verifying (3.13) for interpolation-like operators $I_{h}$ based on orthogonalized B-splines. We consider a special choice for $\mathrm{I}_{h}(3.8)$ : let $\widetilde{\eta}$ and $A$ be the B-splines of order $M \geq 1$ and $N \geq 1$, respectively. Define $\eta$ by

$$
\widehat{\eta}(\sigma):=\frac{\widehat{\widetilde{\eta}}(\sigma)}{\mathbf{a}(\sigma)}=\frac{1}{\sqrt{2 \pi}} \frac{\operatorname{sinc}^{M}(\sigma / 2)}{\mathbf{a}(\sigma)},
$$

where

$$
\mathbf{a}(\sigma)=\sum_{\ell \in \mathbb{Z}} a_{\ell} \mathrm{e}^{-\imath \ell \sigma} \quad \text { with } a_{\ell}=\int_{\mathbb{R}} \widetilde{\eta}(s) A(\ell-s) \mathrm{d} s .
$$

Note that $\mathbf{a}$ is a positive even real trigonometric polynomial with $\mathbf{a}(0)=1$; see Appendix C.1. Further, $\eta$ and $A$ are dual functions; that is,

$$
\langle\eta(\cdot-k), A(\cdot)\rangle=\delta_{k, 0} ;
$$

see Appendix C.2. Especially,

$$
\left(I-\mathrm{I}_{h}\right)\left(I-\widetilde{\mathrm{I}}_{h}\right)=I-\mathrm{I}_{h},
$$

where $\widetilde{\mathrm{I}}_{h} u(s):=h^{-1} \sum_{k \in \mathbb{Z}}\left\langle u, \widetilde{\eta}_{h}\left(\cdot-s_{k}\right)\right\rangle A_{h}\left(s-s_{k}\right)$. 
As in (3.16) we obtain

$$
\left\|\mathrm{I}_{h} u-u\right\|_{-1 / 2} \leq\|u\|_{\alpha-1 / 2} \sup _{v \in H^{1 / 2}(\mathbb{R})} \frac{\left\|\mathrm{I}_{h}^{*} v-v\right\|_{1 / 2-\alpha}}{\|v\|_{1 / 2}}, \quad 0 \leq \alpha \leq 1 / 2 .
$$

Accordingly, we have to investigate the approximation power of the $L^{2}$-adjoint operator $\mathrm{I}_{h}^{*} u(s)=h^{-1} \sum_{k \in \mathbb{Z}}\left\langle u, A_{h}\left(\cdot-s_{k}\right)\right\rangle \eta_{h}\left(s-s_{k}\right)$ which is done in Appendix C.3. By (C.3),

$$
\left\|\mathrm{I}_{h} u-u\right\|_{-1 / 2} \lesssim h^{\alpha}\|u\|_{\alpha-1 / 2}, \quad 0 \leq \alpha \leq 1 / 2 .
$$

The range $\alpha>1 / 2$ we approach as in (3.18) with the help of (4.4):

$$
\left\|\mathrm{I}_{h} u-u\right\|_{-1 / 2} \lesssim h^{1 / 2}\left\|\widetilde{\mathrm{I}}_{h} u-u\right\|_{L^{2}(\mathbb{R})} .
$$

Applying Theorem A.2 to the above right-hand side implies (3.13) with

$$
\alpha_{\mathrm{I}}= \begin{cases}3 / 2 & : \quad N=1, \\ 5 / 2 & : \quad N \geq 2 .\end{cases}
$$

The reconstruction filter belonging to $\mathrm{I}_{h}$ considered in this section is

$$
v(s)=\frac{1}{\pi \sqrt{2 \pi}} \int_{0}^{\infty} \sigma \frac{\operatorname{sinc}^{M}(\sigma / 2)}{\mathbf{a}(\sigma)} \widehat{B}(\sigma) \cos (s \sigma) \mathrm{d} \sigma .
$$

To find an explicit representation of a poses no problem since $a_{\ell}=\mathcal{B}(\ell)$, where $\mathcal{B}$ is the B-spline of order $M+N$. So, $a_{\ell} \in \mathbb{Q}$ can be found by the B-spline recursion or explicit representations of B-splines. Nevertheless, $v$ cannot be evaluated explicitly in general. However, the needed values of $v$ at integers can be computed numerically to any desired accuracy.

Example 4.1. Let $M=1, N=2$, and $B(s)=\operatorname{sinc}(\pi s)$. Then, $\mathbf{a}(\sigma)=\frac{3}{4}+$ $\frac{1}{4} \cos (\sigma)$ and

$$
v(s)=\frac{4}{\pi^{2}} \int_{0}^{\pi} \frac{\sin (\sigma / 2) \cos (s \sigma)}{3+\cos (\sigma)} \mathrm{d} \sigma
$$

Using this filter in the FBA together with piecewise linear interpolation in $\mathrm{I}_{h}$ yields

$$
\left\|\widetilde{f}_{\mathrm{FB}}-f\right\|_{L^{2}(\Omega)} \lesssim h^{\min \{5 / 2, \alpha\}}\|f\|_{\alpha} \quad \text { for } f \in H_{0}^{\alpha}(\Omega), \alpha>0,
$$

since band-limited interpolation (B.4) is considered for $E_{h}\left(\beta_{\max }=\tau_{\max }>3\right)$.

Remark 4.2. The biorthogonalization procedure (4.1) is the same procedure used in the construction of orthogonal spline wavelets; see Lemarié [12]. The connection between wavelets and reconstruction filters can even be extended to increase $\alpha_{\mathrm{I}}$. Choosing $A$ to be a B-spline of order $N$ and $\eta$ to be a suitable compactly supported dual scaling function (see Cohen, Daubechies, and Feauveau [4]) yields an operator $\mathrm{I}_{h}$ with an $\alpha_{\mathrm{I}}$ increasing with $N$. The needed approximation properties of $\mathrm{I}_{h}$ and $\mathrm{I}_{h}^{\star}$ are reported, for instance, by Dahmen [5, Prop. 5.1]. 
5. Verifying (3.13) for interpolation-like operators $I_{h}$ based on B-splines. Our analysis presented so far does not cover operators $I_{h}$ where $\eta$ and $A$ are B-splines of order $M$ and $N$, respectively. We will now investigate this situation.

Let $E_{h}$ and $\mathrm{I}_{h}$ be defined as earlier with respect to $\epsilon, B, \eta$, and $A$. Moreover, let $\mathbf{a}$ be given as in (4.2); however, $\widetilde{\eta}$ is replaced by $\eta$. Further, define the operator $\mathbf{A}_{h}: L^{2}(\mathbb{R}) \rightarrow L^{2}(\mathbb{R}), h>0$, by $\widehat{\mathbf{A}_{h} u}(\sigma):=\mathbf{a}(h \sigma) \widehat{u}(\sigma)$. Note that $\widehat{\mathbf{A}_{h}^{-1} u}(\sigma)=$ $\widehat{u}(\sigma) / \mathbf{a}(h \sigma)$. Now the key observation is that

$$
\widetilde{f}_{\mathrm{FB}}=\frac{1}{2 \pi} \mathbf{R}^{*} \mathrm{I}_{h} \Lambda E_{h} \mathbf{R} f=\frac{1}{2 \pi} \mathbf{R}^{*} \mathrm{I}_{h} \mathbf{A}_{h}^{-1} \Lambda \mathbf{A}_{h} E_{h} \mathbf{R} f .
$$

Consequently, we have to study the approximation powers of the products $\mathbf{A}_{h} E_{h}$ and $\mathrm{I}_{h} \mathbf{A}_{h}^{-1}$. The latter product is exactly the operator $\mathrm{I}_{h}$ studied in the former section. Hence,

$$
\left\|\mathrm{I}_{h} \mathbf{A}_{h}^{-1}-I\right\|_{H^{\alpha-1 / 2}(\mathbb{R}) \rightarrow H^{-1 / 2}(\mathbb{R})} \lesssim h^{\alpha}, \quad 0 \leq \alpha \leq \alpha_{\mathrm{I}}=\left\{\begin{array}{l}
3 / 2: N=1 \\
5 / 2: N \geq 2 .
\end{array}\right.
$$

The product $\mathbf{A}_{h} E_{h}$ requires a little bit more attention. We begin with

$$
\left\|\mathbf{A}_{h} E_{h} u-u\right\|_{\tau} \lesssim\left\|E_{h} u-u\right\|_{\tau}+\left\|\mathbf{A}_{h} u-u\right\|_{\tau} .
$$

In view of (3.12) and (C.2) we obtain

$$
\left\|\mathbf{A}_{h} E_{h} u-u\right\|_{\tau} \lesssim h^{\beta-\tau}\|u\|_{\beta}, \quad u \in H_{0}^{\beta}(-1,1),
$$

for $\beta_{\min } \leq \beta \leq \min \left\{\beta_{\max }, 2+\tau\right\}, 0 \leq \tau \leq \beta, \tau \leq \tau_{\max }$. The parameters $\beta_{\min }, \beta_{\max }$, and $\tau_{\max }$ correspond to $E_{h}$.

Theorem 3.7 holds accordingly, however, with the following restrictions on $\alpha$ :

$$
\max \left\{0, \beta_{\min }-1 / 2\right\}<\alpha \leq \min \left\{\alpha_{\mathrm{I}}, 2, \beta_{\max }-1 / 2, \tau_{\max }-1 / 2\right\} ;
$$

that is, the maximal convergence order cannot exceed 2 which is a tribute to the operator $\mathbf{A}_{h}$ in front of $E_{h}$.

Example 5.1. Using the Shepp-Logan filter $\left(\eta=\chi_{[-1 / 2,1 / 2[}, B(s)=\operatorname{sinc}(\pi s)\right)$ in the FBA together with piecewise linear interpolation in $\mathrm{I}_{h}$ ( $A$ is the linear B-spline) yields

$$
\left\|\widetilde{f}_{\mathrm{FB}}-f\right\|_{L^{2}(\Omega)} \lesssim h^{\min \{2, \alpha\}}\|f\|_{\alpha} \quad \text { for } f \in H_{0}^{\alpha}(\Omega), \alpha>0,
$$

when $\epsilon$ is either an even, compactly supported, and normalized $L^{2}$-function (Theorem B.2) or the Dirac distribution (Theorem B.4).

6. Numerical illustrations. We provide numerical experiments to illustrate the convergence results proved in the former sections. Especially, we will see that the convergence rates saturate indeed at the given bounds.

To this end we need an $f \in L^{2}(\Omega)$ with a prescribed Sobolev order and with an analytically computable Radon transform. We favor the following construction. Let $p_{n}$ be defined by $p_{n}(x)=\left(1-\|x\|^{2}\right)^{n},\|x\| \leq 1$, and $p_{n}(x)=0$, otherwise. We have that $p_{n} \in H_{0}^{\alpha}(\Omega)$ for any $\alpha<n+1 / 2$. The function $f$ for the first numerical experiment is then given by

$$
f(x):=\sum_{k=1}^{3} d_{k} p_{3}\left(U_{k}\left(x-b_{k}\right)\right) \in H_{0}^{\alpha}(\Omega) \text { for any } \alpha<7 / 2,
$$



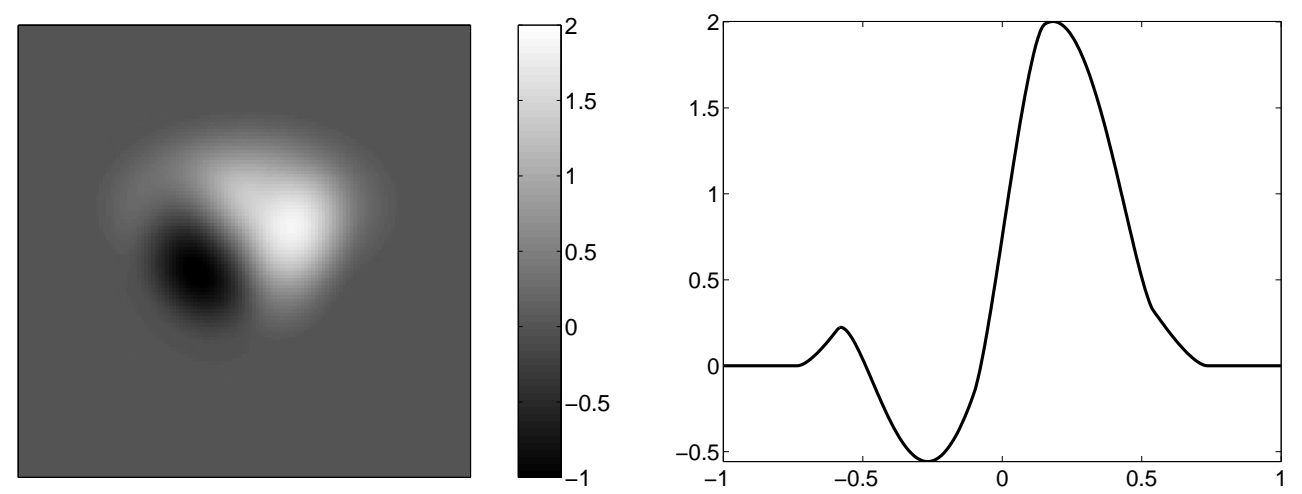

Fig. 2. The function $f$ from (6.1) (left) and its cross section $f(\cdot, 0)$ (right).

where $d_{1}=1, d_{2}=-1.5, d_{3}=1.5$, and $b_{1}=(0.22,0)^{t}, b_{2}=(-0.22,0)^{t}, b_{3}=(0,0.2)^{t}$. Further, $U_{k}=U\left(\varphi_{k}, \delta_{k}, \gamma_{k}\right), k=1,2,3$, with

$$
U(\varphi, \delta, \gamma):=\left(\begin{array}{rr}
\cos (\varphi) / \delta & \sin (\varphi) / \delta \\
-\sin (\varphi) / \gamma & \cos (\varphi) / \gamma
\end{array}\right)
$$

and

$$
\begin{array}{lll}
\delta_{1}=0.51, & \gamma_{1}=0.31, & \varphi_{1}=72 \pi / 180, \\
\delta_{2}=0.51, & \gamma_{2}=0.36, & \varphi_{2}=108 \pi / 180, \\
\delta_{3}=0.5, & \gamma_{3}=0.8, & \varphi_{3}=\pi / 2 .
\end{array}
$$

See Figure 2 for a graphical representation of $f$. We reconstructed $f$ on the grid $X_{q}:=\Omega \cap\{(i / q, j / q) \mid-q \leq i, j \leq q\}$ by

$$
f_{\mathrm{FB}, q}(x):=\frac{1}{2 \pi} \mathbf{R}_{3 q}^{*} \mathrm{I}_{1 / q} \Lambda E_{1 / q} \mathbf{R} f(x), \quad x \in X_{q},
$$

where $\mathbf{R}_{p}^{*}$ is defined in (1.6). We have chosen the number of directions (3q) close to its optimal value; see, e.g., Natterer [14, p. 84].

Now we define the relative $\ell^{2}$-reconstruction error $e$ by

$$
e(q):=\left(\sum_{x \in X_{q}}\left(f_{\mathrm{FB}, q}(x)-f(x)\right)^{2} / \sum_{x \in \mathcal{X}_{q}} f(x)^{2}\right)^{1 / 2} .
$$

In Figure 3 we plotted $e$ as the function of $q \in\{25,50,75,100,125,150,175,200\}$ on a double logarithmic scale with respect to three different settings in the FBA:

- The Shepp-Logan filter with nearest-neighbor interpolation (Example 3.8). Here, the expected and observed convergence rate is $e(q) \sim q^{-3 / 2}$; see the dot-dashed line marked with $\square$.

- The Shepp-Logan filter with piecewise linear interpolation (Example 5.1). Here, the expected and observed convergence rate is $e(q) \sim q^{-2}$; see the dashed line marked with $\triangle$.

- The modified Shepp-Logan filter with piecewise linear interpolation (Example 4.1). Here, the expected and observed convergence rate is $e(q) \sim q^{-5 / 2}$; see the solid line marked with $\mathrm{O}$. We also plotted an auxiliary curve decaying exactly like $q^{-5 / 2}$ (solid line in light gray). 


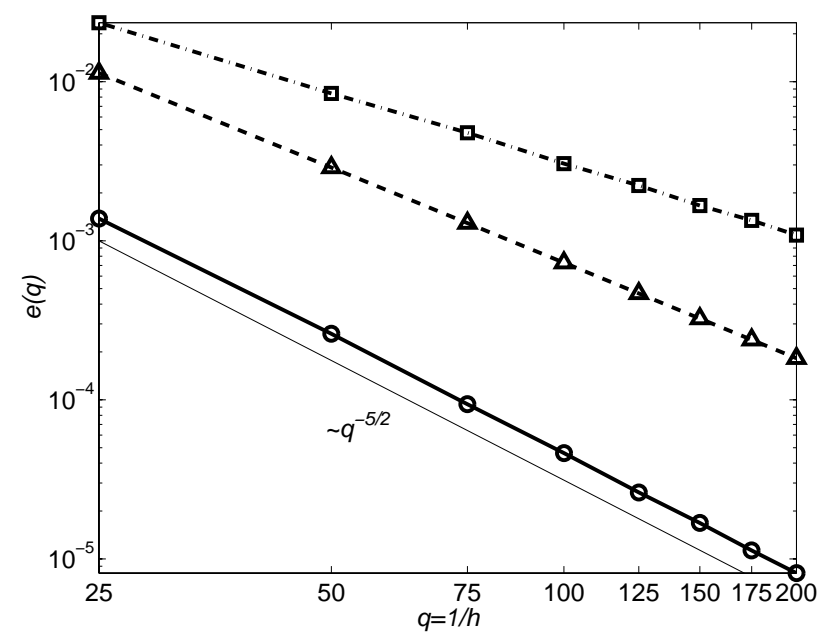

FIG. 3. The relative $\ell^{2}$-errors e (6.3) for reconstructing $f$ (6.1) by the FBA using the SheppLogan filter with nearest-neighbor interpolation (dot-dashed with $\square$ ), the Shepp-Logan filter with piecewise linear interpolation (dashed with $\triangle$ ), and the modified Shepp-Logan filter with piecewise linear interpolation (solid with $\mathrm{O}$ ). The auxiliary solid line indicates exact decay $q^{-5 / 2}$.

In light of the computational experiments we may conclude that our bounds for the maximal convergence orders cannot be improved (at least for the settings underlying the experiments).

Next, we present the relative $\ell^{2}$-errors in reconstructing the Shepp-Logan head phantom; see Figure 4. The Shepp-Logan head phantom $f^{\mathrm{SL}}$ simulates the geometry and the density relations in a human skull. It consists of superimposed indicator functions of ellipses. Hence, $f^{\mathrm{SL}} \in H_{0}^{\alpha}(\Omega)$ for any $\alpha<1 / 2 .{ }^{4}$ We therefore expect and observe $e(q) \sim q^{-1 / 2}$ for all three settings from above.

Both experiments agree completely with our theoretical results, although a discretization of the backprojection operator was not investigated. With our last experiment we justify this simplification once more by considering a setting which might cause trouble in a convergence analysis including the discrete backprojection operator.

The function to be reconstructed consists of indicator functions of two rectangles $\mathcal{R}_{1}$ and $\mathcal{R}_{2}$ :

$$
f(x):=\chi_{\mathcal{R}_{1}}(x)+0.5 \chi_{\mathcal{R}_{2}}(x)
$$

with

$$
\mathcal{R}_{1}:=[-2 / 5,2 / 5] \times[-3 / 5,3 / 5]
$$

and $(U$ as in $(6.2))$

$$
\mathcal{R}_{2}:=\left\{x \in \mathbb{R}^{2} \mid U(\pi / 3,0.7,0.4)(x-b) \in[-1,1]^{2}\right\}, \quad b=(-0.1,-0.1)^{t} ;
$$

see Figure 5 (left). Note that $f$ is in $H_{0}^{\alpha}(\Omega)$ for any $\alpha<1 / 2$. So what is the difference to the Shepp-Logan head phantom? While the fact that $\mathbf{R} f$ as a function

\footnotetext{
${ }^{4}$ In general, picture densities in medical imaging can be considered elements in $H_{0}^{\alpha}(\Omega)$ with $\alpha<1 / 2$ but close to $1 / 2$; see Natterer [14, pp. 92 ff.].
} 

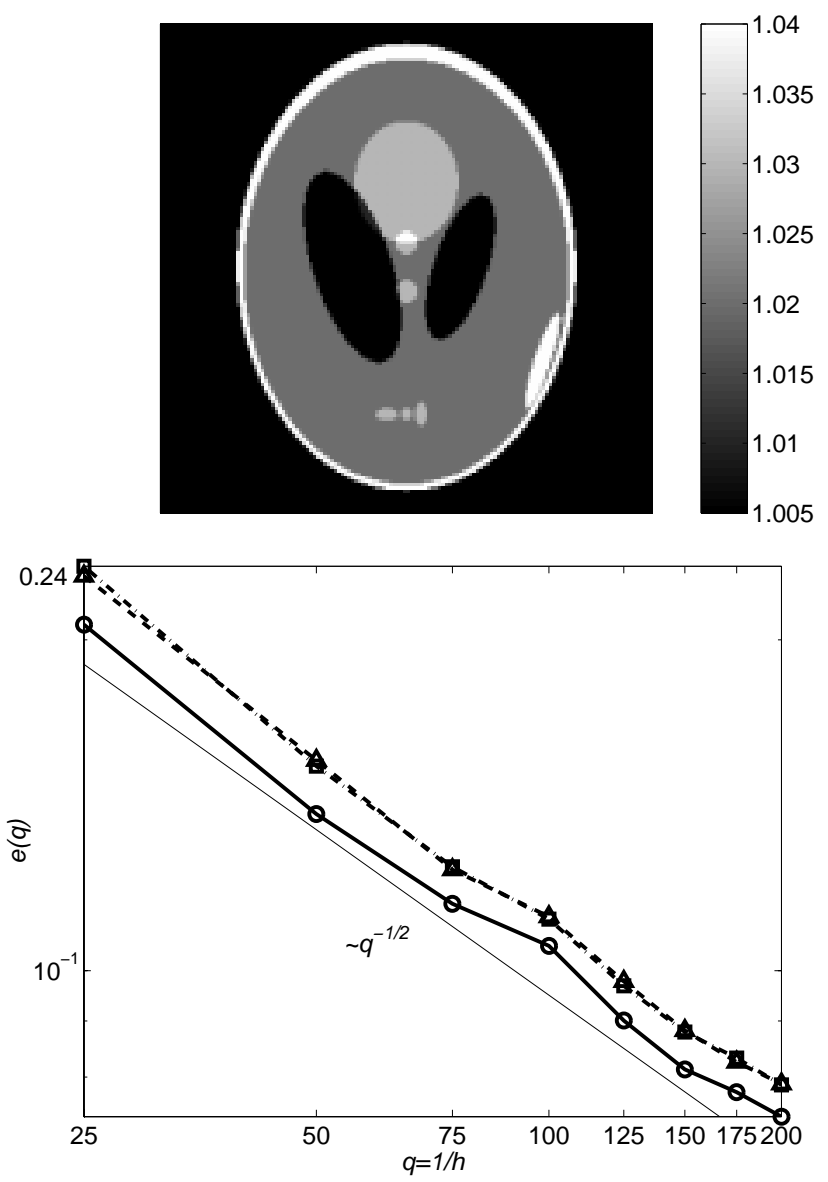

FIG. 4. Top: head phantom due to Shepp-Logan [21]. Bottom: the relative $\ell^{2}$-errors e (6.3) for reconstructing the Shepp-Logan phantom by the FBA using the Shepp-Logan filter with nearestneighbor interpolation (dot-dashed with $\square$ ), the Shepp-Logan filter with piecewise linear interpolation (dashed with $\triangle$ ), and the modified Shepp-Logan filter with piecewise linear interpolation (solid with $\mathrm{O})$. The auxiliary solid line indicates exact decay $q^{-1 / 2}$.

of two variables lies in $H_{0}^{\beta}(-1,1) \widehat{\otimes} L^{2}(0, \pi)$ implies that the functions of one variable $\mathbf{R} f(\cdot, \vartheta)$ lie in $H_{0}^{\beta}(-1,1)$ for almost all $\vartheta$, there may be a null set of exceptional angles $\vartheta$, where $\mathbf{R} f(\cdot, \vartheta)$ has less Sobolev regularity. For $f$ given in (6.4) we have that $\mathbf{R} f$ is in $H_{0}^{\beta}(-1,1) \widehat{\otimes} L^{2}(0, \pi)$ for any $\beta<1$, but there exist four angles $\vartheta$, where $\mathbf{R} f(\cdot, \vartheta)$ is less smooth. Indeed,

$$
\mathbf{R} f(\cdot, \vartheta) \in H_{0}^{\alpha}(-1,1), \alpha<1 / 2, \quad \text { for } \vartheta \in\{0, \pi / 3, \pi / 2,5 \pi / 6\} ;
$$

see Figure 5 (right). The bound on $\alpha$ is maximal (there are no such pathological angles for the Shepp-Logan head phantom; however, one expects such angles in real measurements from medical imaging).

In Figure 6 we plotted the relative reconstruction error (6.3) for the same $q$-values as before. Please note that the discrete Radon data for all $q$ contain integrals over lines which run along the boundary of $\mathcal{R}_{1}$. Further, all used reconstruction grids $x_{q}$ have sufficiently many points on the boundary of $\mathcal{R}_{1}$; indeed, the cardinality of 

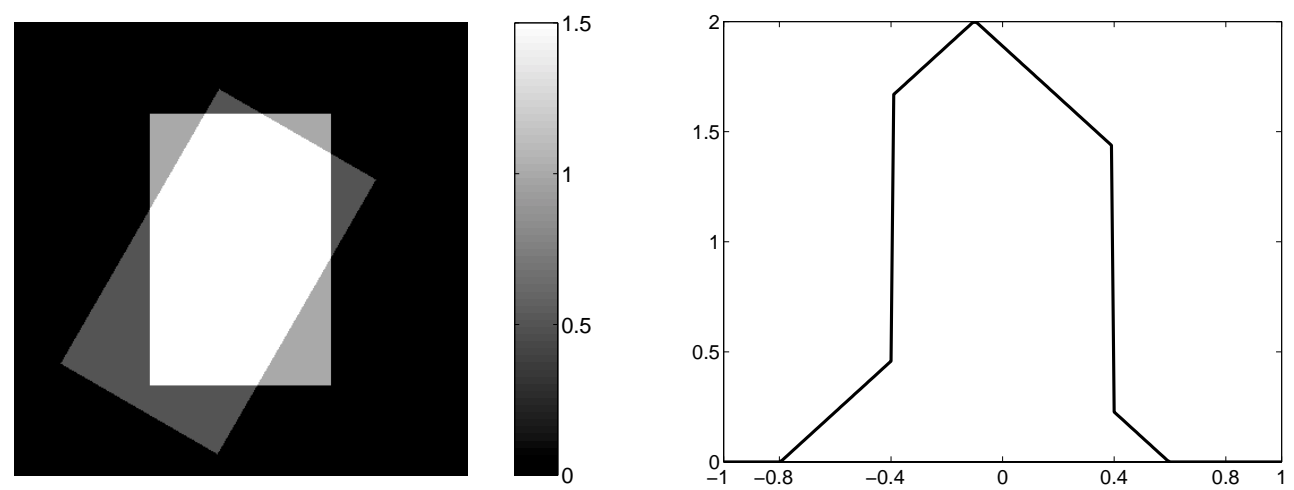

FIG. 5. The function $f$ from (6.4) (left) and its projection $\mathbf{R} f(\cdot, 0)$ (right). The jumps of $\mathbf{R} f(\cdot, 0)$ in $\pm 2 / 5$ are clearly visible.

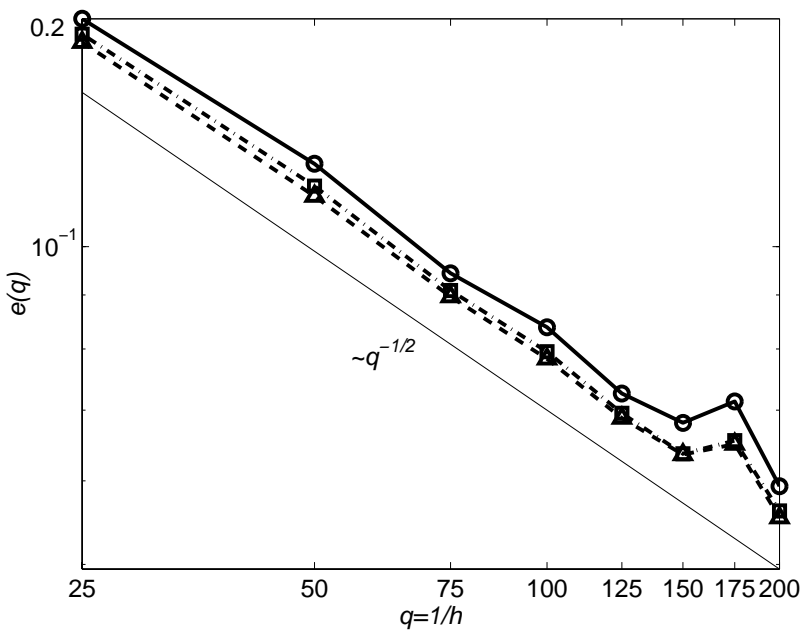

FIG. 6. The relative $\ell^{2}$-errors e (6.3) for reconstructing $f$ (6.4) by the FBA using the SheppLogan filter with nearest-neighbor interpolation (dot-dashed with $\square$ ), the Shepp-Logan filter with piecewise linear interpolation (dashed with $\triangle$ ), and the modified Shepp-Logan filter with piecewise linear interpolation (solid with $\mathrm{O}$ ). The auxiliary solid line indicates exact decay $q^{-1 / 2}$.

$x_{q} \cap \partial \mathcal{R}_{1}$ increases like $\mathrm{O}(q)$.

We observe that even "pathological" projections do not deteriorate the convergence rate obtained by using the continuous backprojection operator for the analysis.

Appendix A. Proof of (3.12) for interpolation-like operators $E_{h}$ based on B-splines. We consider $E_{h}$ as defined in (3.6) where $B$ is the cardinal B-spline of order $N \geq 1$; that is, $B$ is the $N$-fold convolution of $\chi_{[-1 / 2,1 / 2]}$ with itself. The functional $\epsilon \in H_{0}^{-\beta_{\min }}(\mathbb{R}), \beta_{\min } \geq 0$, is supposed to be even, compactly supported in $\square=[-a, a], a>0$, and normalized by $\langle 1, \epsilon\rangle=1$ where $\langle\cdot, \cdot\rangle$ denotes the duality pairing in $H^{\beta_{\min }}(\square) \times H_{0}^{-\beta_{\min }}(\square)$.

The techniques we use below are standard in approximation theory, yet we are not aware of any reference suitable for our setting; however, see Aubin [1, sect. 8.6].

First, we show that $E_{h}$ reproduces affine linear functions if $N \geq 2$. 
Lemma A.1. If $N \geq 2$, then $E_{h} p=p$ for any $p \in \Pi_{1}$. For $N=1 E_{h}$ reproduces only constants.

Proof. Note that the action of $E_{h}$ on $p$ is well defined since $\epsilon$ has compact support. Constants are preserved by $\langle 1, \epsilon(\cdot-k)\rangle=1$ and $\sum_{k \in \mathbb{Z}} B(s-k)=1$; see, e.g., Schoenberg [20, p. 16]. Let $p(s)=s$; then $\langle p(\cdot), \epsilon(\cdot-k)\rangle=k$ due to the evenness of $\epsilon$. By $s=\sum_{k \in \mathbb{Z}} k B(s-k), N \geq 2$ (see, e.g., Schoenberg [20, p. 16]), we are done.

Theorem A.2. Let $\beta_{\min } \leq \beta \leq \min \{2, N\}, \tau<N-1 / 2$, and $0 \leq \tau \leq \beta$. Then,

$$
\left\|E_{h} u-u\right\|_{\tau} \lesssim h^{\beta-\tau}\|u\|_{\beta} \quad \text { as } h \rightarrow 0 .
$$

Proof. We restrict the proof to $N \geq 2$, and we show first a local version of the approximation property. Therefore, let $\square_{h, k}:=h(\square+2 a k)$ for $k \in \mathbb{Z}$. We will rely on the Bramble-Hilbert-like estimate (A.1): there is an affine linear function $P=P(u)$ such that

$$
\|u-P\|_{H^{\tau}\left(\square_{h, k}\right)} \lesssim h^{\beta-\tau}\|u\|_{H^{\beta}\left(\square_{h, k}\right)}, \quad 0 \leq \tau \leq \beta \leq 2 .
$$

For $\tau=0$, (A.1) reduces to the original estimate by Bramble and Hilbert [2]. For positive real $\tau$, see Dupont and Scott [7, Thm. 6.1] or Brenner and Scott [3, Lem. 4.3.8]. By Lemma A.1 and (A.1) we have

$$
\left\|E_{h} u-u\right\|_{H^{\tau}\left(\square_{h, k}\right)} \lesssim\left\|E_{h}(u-p)\right\|_{H^{\tau}\left(\square_{h, k}\right)}+h^{\beta-\tau}\|u\|_{H^{\beta}\left(\square_{h, k}\right)} .
$$

Let $\mathcal{J}_{h, k}:=\left\{r \in \mathbb{Z} \mid \operatorname{supp} B_{h}\left(\cdot-s_{r}\right) \cap \square_{h, k} \neq \emptyset\right\}$. The cardinality of $\mathcal{J}_{h, k}$ neither depends on $h$ nor on $k$. We proceed with

$$
\begin{aligned}
\left\|E_{h}(u-p)\right\|_{H^{\tau}\left(\square_{h, k}\right)} & \lesssim \sum_{r \in \mathcal{J}_{h, k}} h^{-1}\left|\left\langle u-P, \epsilon_{h}\left(\cdot-s_{r}\right)\right\rangle\right|\left\|B_{h}\left(\cdot-s_{r}\right)\right\|_{H^{\tau}(\mathbb{R})} \\
& \lesssim h^{-\tau} \sum_{r \in \mathcal{J}_{h, k}} h^{-1 / 2}\left|\left\langle u-P, \epsilon_{h}\left(\cdot-s_{r}\right)\right\rangle\right| .
\end{aligned}
$$

From the proof of Lemma 5.2 by Dahmen, Prössdorf, and Schneider [6] we know that

$$
\left|\left\langle u-P, h^{-1 / 2} \epsilon_{h}\left(\cdot-s_{r}\right)\right\rangle\right|^{2} \lesssim\|u-P\|_{L^{2}\left(\square_{h, r}\right)}^{2}+h^{2 \beta_{\min }}\|u-P\|_{H^{\beta_{\min }\left(\square_{h, r}\right)}}^{2},
$$

which, by (A.1), gives

$$
\begin{aligned}
\left\|E_{h} u-u\right\|_{H^{\tau}\left(\square_{h, k}\right)} & \lesssim h^{\beta-\tau} \sum_{r \in \mathcal{J}_{h, k}}\|u\|_{H^{\beta}\left(\square_{h, r}\right)} \lesssim h^{\beta-\tau}\left(\sum_{r \in \mathcal{J}_{h, k}}\|u\|_{H^{\beta}\left(\square_{h, r}\right)}^{2}\right)^{1 / 2} \\
& \lesssim h^{\beta-\tau}\|u\|_{H^{\beta}\left(\widetilde{\square}_{h, k}\right)},
\end{aligned}
$$

where $\widetilde{\square}_{h, k}:=\bigcup_{r \in \mathcal{J}_{h, k}} \square_{h, r}$. Thus,

$$
\left\|E_{h} u-u\right\|_{H^{\tau}\left(\square_{h, k}\right)} \lesssim h^{\beta-\tau}\|u\|_{H^{\beta}\left(\widetilde{\square}_{h, k}\right)} .
$$

Squaring both sides of the latter local approximation property and summing over $k \in \mathbb{Z}$ yield finally the stated global approximation property.

Summary. The above theorem covers especially the cases $\epsilon=\delta$ (Dirac distribution), where $\beta_{\min }>1 / 2$, and $\epsilon \in L^{2}(\square)$ being even with $\int_{\square} \epsilon(s) \mathrm{d} s=1$, where $\beta_{\min }=0$. Hence, for both latter cases (3.12) holds with $\beta_{\max }=2$ and $\tau_{\max }<N-1 / 2$. 
Appendix B. Proof of (3.12) for interpolation-like operators $E_{\boldsymbol{h}}$ based on the sinc-function. We consider $E_{h}$ as defined in (3.6) where $B$ is the sinus cardinalis; that is, $B(x):=\operatorname{sinc}(\pi x)$, where $\operatorname{sinc}(x)=\sin (x) / x, x \neq 0$, and $\operatorname{sinc}(0)=$ 1. Further, $\epsilon \in L^{1}(\mathbb{R}) \cap L^{2}(\mathbb{R})$ is an even function with compact support and a normalized mean value, $\int \epsilon(x) \mathrm{d} x=1$. Here, $\langle\cdot, \cdot\rangle$ denotes the $L^{2}(\mathbb{R})$-inner product.

First we bound $E_{h}$ uniformly in $h$.

Lemma B.1. (a) The operators $E_{h}: L^{2}(\mathbb{R}) \rightarrow L^{2}(\mathbb{R}), h>0$, are uniformly bounded in $h$.

(b) Let $w$ be in $L^{2}(\mathbb{R})$ with supp $\widehat{w} \subset[-\pi / h, \pi / h]$. Then, we have the inverse estimate $\|w\|_{\alpha} \leq 2^{\alpha / 2} \pi^{\alpha} h^{-\alpha}\|w\|_{L^{2}(\mathbb{R})}$ for $0<h \leq \pi$ and any $\alpha \geq 0$.

Proof. (a) Set $\square_{h, k}=h(\operatorname{supp} \epsilon+k)$. The $L^{2}(\mathbb{R})$-orthogonality of $\left\{B_{h}\left(\cdot-s_{k}\right)\right\}_{k \in \mathbb{Z}}$ gives

$$
\left\|E_{h} u\right\|_{L^{2}(\mathbb{R})}^{2}=h^{-1} \sum_{k \in \mathbb{Z}}\left|\left\langle u, \epsilon_{h}\left(\cdot-s_{k}\right)\right\rangle\right|^{2} \leq \sum_{k \in \mathbb{Z}}\|u\|_{L^{2}\left(\square_{h, k}\right)}^{2}\|\epsilon\|_{L^{2}(\mathbb{R})}^{2} \lesssim\|u\|_{L^{2}(\mathbb{R})}^{2} .
$$

(b) The inverse estimate results from a straightforward estimate of $\|w\|_{\alpha}^{2}$ taking into account the compact support of $\widehat{w}$.

After the above preparatory results we are able to prove the claimed convergence estimate.

TheOREm B.2. Let $0 \leq \tau \leq \beta, \beta-\tau \leq 2$. Under the assumptions from above we have that

$$
\left\|E_{h} u-u\right\|_{\tau} \lesssim h^{\beta-\tau}\|u\|_{\beta} \quad \text { as } h \rightarrow 0
$$

Proof. Define an auxiliary operator $P_{h}: L^{2}(\mathbb{R}) \rightarrow L^{2}(\mathbb{R})$ by

$$
\widehat{P_{h} w}(\xi):=\chi_{\square_{h}}(\xi) \widehat{w}(\xi) .{ }^{5}
$$

It is an easy exercise to obtain

$$
\left\|P_{h} u-u\right\|_{\tau} \lesssim h^{\beta-\tau}\|u\|_{\beta} \quad \text { for } 0 \leq \tau \leq \beta<\infty
$$

whenever the right-hand side is finite.

In a first step we consider $\left\|E_{h} P_{h} u-P_{h} u\right\|_{\tau}$. We have

$$
\widehat{E_{h} P_{h} u}(\xi)=\frac{1}{\sqrt{2 \pi}} \chi_{\square_{h}}(\xi) \sum_{k \in \mathbb{Z}}\left\langle P_{h} u, \epsilon_{h}\left(\cdot-s_{k}\right)\right\rangle \mathrm{e}^{-\imath h k \xi}
$$

and

$$
\begin{aligned}
h^{1 / 2}\left\langle P_{h} u, \epsilon_{h}\left(\cdot-s_{k}\right)\right\rangle & =h^{1 / 2} \int_{\square_{h}} \widehat{P_{h} u}(\xi) \widehat{\epsilon_{h}}(\xi) \mathrm{e}^{\imath h k \xi} \mathrm{d} \xi \\
& =\left(\frac{h}{2 \pi}\right)^{1 / 2} \int_{\square_{h}} \widehat{P_{h} u \star \epsilon_{h}}(\xi) \mathrm{e}^{\imath h k \xi} \mathrm{d} \xi,
\end{aligned}
$$

which is the $k$ th Fourier coefficient of $\widehat{P_{h} u \star \epsilon}$. Hence,

$$
\widehat{E_{h} P_{h} u}(\xi)=h^{-1} \chi_{\square_{h}}(\xi) \widehat{P_{h} u \star \epsilon_{h}}(\xi) .
$$

\footnotetext{
${ }^{5}$ Actually, $P_{h}$ is the orthogonal projector onto the closed subspace of band-limited functions with band-width $\pi / h$.
} 
Therefore,

$$
\begin{aligned}
\left\|E_{h} P_{h} u-P_{h} u\right\|_{\tau}^{2} & =\int_{\square_{h}}\left(1+\xi^{2}\right)^{\tau}\left|h^{-1} \widehat{P_{h} u \star \epsilon_{h}}(\xi)-\widehat{P_{h} u}(\xi)\right|^{2} \mathrm{~d} \xi \\
& \lesssim \int_{\square_{h}}\left(1+\xi^{2}\right)^{\tau}|\widehat{u}(\xi)|^{2} M(h \xi) \mathrm{d} \xi
\end{aligned}
$$

where $M(z)=|\widehat{\epsilon}(z)-1 / \sqrt{2 \pi}|^{2}, z \in \mathbb{R}$. As in the proof of Corollary 2.2 one shows that $M(z) \lesssim z^{4}$ using a Taylor expansion of $\widehat{\epsilon}$ about the origin. Now let $0 \leq \beta-\tau \leq 2$. Then,

$$
\begin{aligned}
\left\|E_{h} P_{h} u-P_{h} u\right\|_{\tau}^{2} & \lesssim h^{4} \int_{\square_{h}}\left(1+\xi^{2}\right)^{\beta}|\widehat{u}(\xi)|^{2} \xi^{4-2(\beta-\tau)} \mathrm{d} \xi \\
& \lesssim h^{2(\beta-\tau)}\|u\|_{\beta}^{2} .
\end{aligned}
$$

In the final step we use both statements from Lemma B.1 as well as (B.2) and (B.3):

$$
\begin{aligned}
\left\|E_{h} u-u\right\|_{\tau} \leq\left\|E_{h} u-E_{h} P_{h} u\right\|_{\tau}+\left\|E_{h} P_{h} u-P_{h} u\right\|_{\tau} \\
+\left\|P_{h} u-u\right\|_{\tau} \\
\lesssim h^{-\tau}\left\|u-P_{h} u\right\|_{L^{2}(\mathbb{R})}+h^{\beta-\tau}\|u\|_{\beta} .
\end{aligned}
$$

Applying (B.2) again we conclude with the proof of Theorem B.2.

Remark B.3. The upper bound 2 on $\beta-\tau$ in Theorem B.2 may be relaxed by imposing higher order vanishing moments on $\epsilon$.

Now we investigate band-limited interpolation; that is, $E_{h}$ is defined by

$$
E_{h} u(s)=\sum_{k \in \mathbb{Z}} u\left(s_{k}\right) \operatorname{sinc}\left(\frac{\pi}{h}\left(s-s_{k}\right)\right) .
$$

Theorem B.4. Let $\beta_{\max } \in \mathbb{N}$. Then, for $1 / 2<\beta<\infty, 0 \leq \tau \leq \beta$ with $\beta-\tau \leq \beta_{\max }$, we have that

$$
\left\|E_{h} u-u\right\|_{\tau} \lesssim h^{\beta-\tau}\|u\|_{\beta} \quad \text { as } h \rightarrow 0
$$

whenever $u \in H^{\beta}(\mathbb{R})$ is compactly supported. The constant in the above estimate may depend on $\beta_{\max }$.

Proof. Band-limited interpolation is well defined under the assumptions on $u$. We introduce an auxiliary operator $E_{h}^{(m)}$. To this end let $\epsilon \in L^{2}(\mathbb{R})$ be compactly supported with normalized mean value $\left(\int \epsilon(x) \mathrm{d} x=1\right)$ and vanishing moments up to order $\beta_{\max }\left(\int x^{k} \epsilon(x) \mathrm{d} x=1, k=1, \ldots, \beta_{\max }\right)$. Thus, $\widehat{\epsilon}(0)=1 / \sqrt{2 \pi}$ and $\widehat{\epsilon}^{(\nu)}(0)=0$, $\nu=1, \ldots, \beta_{\max }$. Define $\epsilon^{(m)}(s):=m \epsilon(m s), m \in \mathbb{N}$, and

$$
E_{h}^{(m)} u(s):=h^{-1} \sum_{k \in \mathbb{Z}}\left\langle u, \epsilon_{h}^{(m)}\left(\cdot-s_{k}\right)\right\rangle \operatorname{sinc}\left(\frac{\pi}{h}\left(s-s_{k}\right)\right)
$$

Observe that $E_{h}^{(m)}: L^{2}(\mathbb{R}) \rightarrow L^{2}(\mathbb{R})$ is uniformly bounded in $h$ and $m$; see the proof of Lemma B.1. Hence, we may apply Theorem B.2 to obtain

$$
\left\|E_{h}^{(m)} u-u\right\|_{\tau} \lesssim h^{\beta-\tau}\|u\|_{\beta},
$$


where the constant is bounded in $m$, as a careful inspection of the proof of Theorem B.2 shows. Moreover, the upper bound on $\beta-\tau$ in (B.5) is $\beta_{\max }$ since all derivatives of $\widehat{\epsilon}$ up to order $\beta_{\max }$ vanish about 0; see Remark B.3. By (B.5),

$$
\left\|E_{h} u-u\right\|_{\tau} \lesssim\left\|E_{h} u-E_{h}^{(m)} u\right\|_{\tau}+h^{\beta-\tau}\|u\|_{\beta}
$$

Further,

$$
\left\|E_{h} u-E_{h}^{(m)} u\right\|_{\tau} \leq\left\|\operatorname{sinc}_{h / \pi}\right\|_{\tau} \sum_{k \in \mathcal{J}_{m, h}(u)}\left|u\left(s_{k}\right)-\left\langle u, h^{-1} \epsilon_{h}^{(m)}\left(\cdot-s_{k}\right)\right\rangle\right|
$$

with $\mathcal{J}_{m, h}(u)=\left\{k \in \mathbb{Z} \mid s_{k} \in \operatorname{supp} u\right\} \cup\left\{k \in \mathbb{Z} \mid \operatorname{supp} u \cap h\left(m^{-1} \operatorname{supp} \epsilon+k\right)\right\}$. The set $\mathcal{J}_{m, h}(u)$ is finite and its cardinality is bounded in $m$. So we have that $\lim _{m \rightarrow \infty} \| E_{h} u-$ $E_{h}^{(m)} u \|_{\tau}=0$, and the stated estimate is readily seen from (B.6).

Summary. The band-limited interpolation-like operators considered in Theorem B.2 satisfy (3.12) with $\beta_{\min }=0, \beta_{\max }=2+\tau$, and any $\tau_{\max }<\infty$. For the band-limited interpolation (B.4) we have (3.12) with $\beta_{\min }>1 / 2$ and any positive $\tau_{\max }$ and any fixed $\beta_{\max }>1 / 2$.

Appendix C. Complement to section 4. This appendix is devoted to the proof of various auxiliary results from section 4 . Throughout this appendix let $\widetilde{\eta}$ and $A$ be B-splines of order $M \geq 1$ and $N \geq 1$, respectively. Further, let $\eta$ be defined by (4.1).

C.1. The trigonometric polynomial a. Recall that

$$
\mathbf{a}(\sigma)=\sum_{\ell \in \mathbb{Z}} a_{\ell} \mathrm{e}^{-\imath \ell \sigma} \quad \text { with } a_{\ell}=\int_{\mathbb{R}} \widetilde{\eta}(s) A(\ell-s) \mathrm{d} s .
$$

Since $\widetilde{\eta}$ and $A$ are even, so are $\left\{a_{\ell}\right\}_{\ell \in \mathbb{Z}}$ and $\mathbf{a}$. By $\sum_{\ell \in \mathbb{Z}} A(\cdot-\ell)=1$ and $\int \widetilde{\eta}(s) \mathrm{d} s=1$ (see, e.g., Schoenberg [20, p. 16 and p. 2]), we have that $\mathbf{a}(0)=1$. In the remainder of this appendix we verify that $\mathbf{a}$ has no zeros. Then we have established all properties of a claimed and needed in section 4 .

Straightforward calculations reveal that the $a_{\ell}$ 's are the Fourier coefficients of the $2 \pi$-periodic function $2 \pi \sum_{k \in \mathbb{Z}} \widehat{\widetilde{\eta}}(\sigma+2 \pi k) \widehat{A}(\sigma+2 \pi k)$. Hence,

$$
\mathbf{a}(\sigma)=2 \pi \sum_{k \in \mathbb{Z}} \widehat{\widetilde{\eta}}(\sigma+2 \pi k) \widehat{A}(\sigma+2 \pi k)=\sum_{k \in \mathbb{Z}} \operatorname{sinc}^{M+N}(\sigma / 2+\pi k) .
$$

If $M+N$ is even, a clearly has no zeros because there is no $\sigma$ such that $\operatorname{sinc}^{M+N}(\sigma / 2+$ $\pi k)=0$ for all $k \in \mathbb{Z}$. It remains to investigate the odd case $M+N=2 L+1, L \in \mathbb{N}$. We factorize a according to

$$
\mathbf{a}(\sigma)=\sin ^{2 L}(\sigma / 2) \boldsymbol{\Sigma}_{2 L+1}(\sigma) \quad \text { with } \boldsymbol{\Sigma}_{2 L+1}(\sigma):=\sum_{k \in \mathbb{Z}} \frac{(-1)^{k}}{(\sigma / 2+\pi k)^{2 L+1}} .
$$

As multiples of $2 \pi$ are not zeros of a it suffices to show that $\boldsymbol{\Sigma}_{2 L+1}$ has no zeros in ] $0,2 \pi[$. Separating even from odd indices we find

$$
\boldsymbol{\Sigma}_{2 L+1}(\sigma)=2^{-(2 L+1)}\left(S_{2 L+1}(\sigma / 4)-S_{2 L+1}(\sigma / 4+\pi / 2)\right),
$$

where $S_{l}(\sigma):=\sum_{k \in \mathbb{Z}}(\sigma+\pi k)^{-l}, l \geq 2$. Observe that $S_{2 l}(\sigma)>0, l \in \mathbb{N}$. Now,

$$
\left.\frac{\mathrm{d}}{\mathrm{d} \sigma} S_{2 L+1}(\sigma)=-(2 L+1) S_{2 L+2}(\sigma)<0, \quad \sigma \in\right] 0,2 \pi[.
$$

Therefore $S_{2 L+1}$ is strongly decreasing in $] 0,2 \pi\left[\right.$ which gives $\boldsymbol{\Sigma}_{2 L+1}>0$ in $] 0,2 \pi[$. 
C.2. Biorthogonality (4.3). By (4.1) and (C.1) we obtain

$$
\begin{aligned}
& \langle\eta(\cdot-k), A(\cdot)\rangle=\int_{\mathbb{R}} \widehat{\eta}(\sigma) \widehat{A}(\sigma) \mathrm{e}^{\imath k \sigma} \mathrm{d} \sigma \\
& \quad=\int_{0}^{2 \pi} \sum_{n \in \mathbb{Z}} \widehat{\eta}(\sigma+2 \pi n) \widehat{A}(\sigma+2 \pi n) \mathrm{e}^{\imath k \sigma} \mathrm{d} \sigma \\
& \quad=\int_{0}^{2 \pi} \frac{1}{\mathbf{a}(\sigma)} \sum_{n \in \mathbb{Z}} \widehat{\widetilde{\eta}}(\sigma+2 \pi n) \widehat{A}(\sigma+2 \pi n) \mathrm{e}^{\imath k \sigma} \mathrm{d} \sigma=\int_{0}^{2 \pi} \frac{\mathrm{e}^{\imath k \sigma}}{2 \pi} \mathrm{d} \sigma,
\end{aligned}
$$

which is (4.3).

C.3. Approximation power of $\mathbf{I}_{\boldsymbol{h}}^{*}$. We are not able to apply Theorem A.2 directly to $I_{h}^{*}$ as $\eta$ from (4.1) does not have compact support in general. Nevertheless, we will show that the approximation power of $\widetilde{I}_{h}^{*}$ carries over to $\mathrm{I}_{h}^{*}$ (for the notation see section 4). Since

$$
\widehat{\mathrm{I}_{h}^{*} u}(\sigma)=\widehat{\mathrm{I}_{h}^{*} u}(\sigma) / \mathbf{a}(h \sigma)
$$

we have that

$$
\left\|u-\mathrm{I}_{h}^{*} u\right\|_{\tau}^{2} \lesssim \int_{\mathbb{R}}\left(1+\sigma^{2}\right)^{\tau}\left|\mathbf{a}(h \sigma) \widehat{u}(\sigma)-\widehat{\mathrm{I}_{h}^{*} u}(\sigma)\right|^{2} \mathrm{~d} \sigma .
$$

Thus,

$$
\left\|u-\mathrm{I}_{h}^{*} u\right\|_{\tau} \lesssim\left\|\mathbf{A}_{h} u-\widetilde{\mathrm{I}}_{h}^{*} \mathbf{A}_{h} u\right\|_{\tau}+\left\|\widetilde{\mathrm{I}}_{h}^{*} \mathbf{A}_{h} u-\widetilde{\mathrm{I}}_{h}^{*} u\right\|_{\tau},
$$

where $\widehat{\mathbf{A}_{h}} u(\sigma)=\mathbf{a}(h \sigma) \widehat{u}(\sigma)$. Theorem A.2 provides

$$
\left\|\mathbf{A}_{h} u-\widetilde{\mathrm{I}}_{h}^{*} \mathbf{A}_{h} u\right\|_{\tau} \lesssim h^{\beta-\tau}\left\|\mathbf{A}_{h} u\right\|_{\beta} \lesssim h^{\beta-\tau}\|u\|_{\beta}
$$

for $0 \leq \beta \leq \min \{2, M\}, \tau<M-1 / 2$, and $0 \leq \tau \leq \beta$. Further, also by Theorem A.2,

$$
\left\|\widetilde{\mathbf{I}}_{h}^{*} \mathbf{A}_{h} u-\widetilde{\mathrm{I}}_{h}^{*} u\right\|_{\tau} \lesssim\left\|\mathbf{A}_{h} u-u\right\|_{\tau}
$$

whenever $0 \leq \tau<M-1 / 2$, for $M \leq 2$, and $0 \leq \tau \leq 2$, otherwise. A Taylor expansion of $\mathbf{a}$ about 0 proves that $|\mathbf{a}(\sigma)-1| \lesssim \sigma^{2}$. Now we may copy the proof of Corollary 2.2 to obtain

$$
\left\|\mathbf{A}_{h} u-u\right\|_{\tau} \lesssim h^{\min \{2, \beta-\tau\}}\|u\|_{\beta}, \quad 0 \leq \tau \leq \beta
$$

Collecting the pieces we find

$$
\left\|u-\mathrm{I}_{h}^{*} u\right\|_{\tau} \lesssim h^{\beta-\tau}\|u\|_{\beta} \quad \text { as } h \rightarrow 0
$$

for $0 \leq \beta \leq \min \{2, M\}, \tau<\min \{2, M-1 / 2\}$, and $0 \leq \tau \leq \beta$. 


\section{REFERENCES}

[1] J.-P. Aubin, Applied Functional Analysis, 2nd ed., Pure Appl. Math., Wiley, New York, 2000.

[2] J. H. Bramble And S. R. Hilbert, Estimation of linear functionals on Sobolev spaces with application to Fourier transforms and spline interpolation, SIAM J. Numer. Anal., 7 (1970), pp. 112-124.

[3] S. C. Brenner And L. R. Scott, The Mathematical Theory of Finite Element Methods, Texts Appl. Math. 15, Springer, New York, 1994.

[4] A. Cohen, I. Daubechies, and J.-C. Feauveau, Biorthogonal bases of compactly supported wavelets, Comm. Pure Appl. Math., 45 (1992), pp. 485-560.

[5] W. Dahmen, Wavelet and Multiscale Methods for Operator Equations, Acta Numer. 6, Cambridge University Press, Cambridge, UK, 1997, pp. 55-228.

[6] W. Dahmen, S. Prössdorf, And R. Schneider, Wavelet approximation methods for pseudodifferential equations I: Stability and convergence, Math. Z., 215 (1994), pp. 583-620.

[7] T. Dupont And L. R. Scott, Polynomial approximation of functions in Sobolev spaces, Math. Comp., 34 (1980), pp. 441-463.

[8] A. Faridani, Praktische Fragen der lokalen Tomographie, Z. Angew. Math. Mech., 70 (1990), pp. T530-T532.

[9] A. Faridani, D. V. Finch, E. L. Ritman, and K. T. Smith, Local tomography II, SiAm J. Appl. Math., 57 (1997), pp. 1095-1127.

[10] A. FARIDANi AND E. L. RitMAn, High-resolution computed tomography from efficient sampling, Inverse Problems, 16 (2000), pp. 635-650.

[11] I. S. Gradshteyn And I. M. Ryzhik, Table of Integrals, Series, and Products, Academic Press, San Diego, 1980.

[12] P. G. Lemarié, Ondelettes à localisation exponentielle, J. Math. Pures Appl. (9), 67 (1988), pp. 227-236.

[13] A. K. Louis and F. NATterer, Mathematical problems in computerized tomography, Proc. IEEE, 71 (1983), pp. 379-389.

[14] F. NATterer, The Mathematics of Computerized Tomography, Wiley, Chichester, 1986.

[15] D. A. Popov, On convergence of a class of algorithms for the inversion of the numerical Radon transform, in Mathematical Problems of Tomography, Transl. Math. Monogr. 81, L. M. Gelfand and S. G. Gindikin, eds., AMS, Providence, R.I., 1990, pp. 7-65.

[16] A. RIEDER, Principles of reconstruction filter design in 2D-computerized tomography, in Radon Transforms and Tomography, Contemp. Math. 278, T. Quinto, L. Ehrenpreis, A. Faridani, F. Gonzales, and E. Grinberg, eds., AMS, Providence, RI, 2001, pp. 201-226.

[17] A. Rieder, R. Dietz, And Th. Schuster, Approximate inverse meets local tomography, Math. Methods Appl. Sci., 23 (2000), pp. 1373-1387.

[18] A. Rieder And Th. Schuster, The approximate inverse in action II: Convergence and stability, Math. Comp., to appear.

[19] W. Rudin, Functional Analysis, 12th ed., Tata McGraw-Hill, New Delhi, India, 1988.

[20] I. J. Schoenberg, Cardinal Spline Interpolation, CBMS-NSF Regional Conf. Ser. in Appl. Math. 12, SIAM, Philadelphia, 1973.

[21] L. A. Shepp And B. F. Logan, The Fourier reconstruction of a head section, IEEE Trans. Nuc. Sci., 21 (1974), pp. 21-43.

[22] K. T. Smith, Reconstruction formulas in computed tomography, Proc. Sympos. Appl. Math., 27 (1982), pp. 7-23.

[23] K. T. Smith and F. Keinert, Mathematical foundations of computed tomography, Appl. Optics, 24 (1985), pp. 3950-3957.

[24] K. T. Smith, D. C. Solmon, And S. C. Wagner, Practical and mathematical aspects of the problem of reconstructing objects from radiographs, Bull. Amer. Math. Soc., 83 (1977), pp. $1227-1270$.

[25] G. Strang And G. J. Fix, An Analysis of the Finite Element Method, Prentice-Hall Series in Automatic Computation, Prentice-Hall, Englewood Cliffs, NJ, 1973. 